\title{
SPANNING SETS FOR AUTOMORPHIC FORMS AND DYNAMICS OF THE FRAME FLOW ON COMPLEX HYPERBOLIC SPACES
}

\author{
TATYANA FOTH AND SVETLANA KATOK
}

\begin{abstract}
Let $G$ be a semisimple Lie group with no compact factors, $K$ a maximal compact subgroup of $G$, and $\Gamma$ a lattice in $G$. We study automorphic forms for $\Gamma$ if $G$ is of real rank one with some additional assumptions, using dynamical approach based on properties of the homogeneous flow on $\Gamma \backslash G$ and a Livshitz type theorem we prove for such a flow. In the Hermitian case $G=S U(n, 1)$ we construct relative Poincaré series associated to closed geodesics on $\Gamma \backslash G / K$ for one-dimensional representations of $K$, and prove that they span the corresponding spaces of cusp forms.
\end{abstract}

\section{INTRODUCTION}

Let $G$ be a real connected semisimple Lie group with no compact factors, $K$ a maximal compact subgroup of $G$, and $\Gamma$ a discrete subgroup of $G$. A general definition of automorphic forms for $\Gamma$ (cf [10] and [3]) depends on a finite-dimensional representation of $K$. It includes classical holomorphic automorphic forms on Fuchsian groups, Maass wave forms, and automorphic forms on bounded symmetric domains. A convenient construction of automorphic forms is that via relative Poincaré series. It allows to "extend" an automorphic form for a subgroup $\Gamma_{0} \subset \Gamma$ to the whole discrete group $\Gamma$. For $\Gamma_{0}=\{e\}$ we obtain Poincaré series, which can be constructed for any smooth absolutely integrable function on $G([1], \S 5)$. For cocompact $\Gamma$ and in some other cases which are discussed in $\$ 1.2$, Poincaré series provide cusp forms. It may happen that a Poincaré series is identically zero. However, it has been shown (see [22], Ch. 3 and [2], Ch. 5) that for holomorphic automorphic forms on a symmetric bounded domain there exist nonzero Poincaré series for suitable weights. It is not easy, however, to construct cusp forms for non-trivial $\Gamma_{0}$, and even less so to prove that a certain collection spans the corresponding space of cusp forms.

Date: November 15, 2018. 
In the case when $G$ is of real rank one and with finite center, and $\Gamma$ is a lattice in $G$, i.e. $\Gamma \backslash G$ has finite Haar measure, we propose a program of constructing spanning sets for cusp forms based on dynamical properties of the homogeneous flow on $\Gamma \backslash G$ and a Livshitz type theorem that we prove for such a flow (Theorem 8). We carry out this program in the case when, in addition, $K$ has non-trivial 1-dimensional representations. In this case the symmetric space $G / K$ is Hermitian of real rank one, and by the classification of symmetric spaces, a complex hyperbolic space

$$
\mathbb{H}_{\mathbb{C}}^{n}=S U(n, 1) / S(U(n) \times U(1))
$$

for some $n \geq 1$. Let $\Gamma$ be a lattice in $S U(n, 1)$, and $\kappa \geq 1$, an integer. For each loxodromic element $\gamma_{0} \in \Gamma$ we construct a relative Poincaré series $\Theta_{\gamma_{0}, \kappa}$ which is a $\mathbb{C}$-valued cusp form in $S_{2(n+1) \kappa}(\Gamma)$ (for exact definitions see $\S 1.1$, $\$ 1.2$ and $\S 6.3$. The main result of this paper is the following theorem.

Theorem 1. The relative Poincaré series $\left\{\Theta_{\gamma_{0}, \kappa}, \gamma_{0} \in \Gamma\right.$ loxodromic $\}$ $\operatorname{span} S_{2(n+1) \kappa}(\Gamma)$.

This is an extension of a result by S. Katok [12 for the classical case of Fuchsian groups $(n=1)$. The method is based on duality between the introduced relative Poincaré series and closed geodesics on $\Gamma \backslash G / K$ (Theorems 11 and 14), dynamical properties of the frame flow on $\Gamma \backslash G$ which imply a Livshitz theorem-type result (Theorem 8), and the existence of a three-dimensional Lie subalgebra associated to the frame flow which enables the harmonic analysis on $\Gamma \backslash G$ that completes the proof. The proof utilizes two essential features of the complex hyperbolic space: real rank one and the presence of the complex structure.

A natural question of finding finite spanning sets for cusp forms has been addressed for the classical $n=1$ case in [14. The main ingredient here was an approximate version of Livshitz Theorem 15] for geodesic flows, based on more subtle dynamical considerations. The same scheme will probably work for complex hyperbolic spaces of all dimensions and will be pursued elsewhere.

The paper is organized as follows. In $\delta$ 1] we review the general definition of automorphic forms and the relative Poincaré series, discuss their convergence and further implications under additional assumptions. \$2 describes the homogeneous flow on $\Gamma \backslash G$ and its relation to the geodesic flow on the unit tangent bundle $S(\Gamma \backslash G / K)$. In $\S 3$ we prove a special Livshitz Theorem for the homogeneous flow on $\Gamma \backslash G$ and describe a program of constructing spanning sets for cusp forms in the real rank one case. In $\S \S 4-8$ we restrict ourselves to the case of the 
complex hyperbolic space, construct relative Poincaré series associated to closed geodesics in $\Gamma \backslash G / K$, and prove Theorem 1 .

We want to express our gratitude to D. Akhiezer for his suggestion to consider a three-dimensional subalgebra associated to the homogeneous flow, which was crucial in the completion of this work. We also would like to thank the Erwin Schroedinger Institute for Mathematical Physics in Vienna, where the second author spent three months working on this paper in 1997 and 1998, for its hospitality and financial support. The research has begun when the second author was partially supported by NSF grant DMS-9404136.

\section{Automorphic Forms on SyMmetriC SPACES OF SEMISIMPle LIE GROUPS}

1.1. Definitions. Let $G$ be a real connected semisimple Lie group with no compact factors ([4], 1.13.12), $\mathfrak{g}$ its Lie algebra, and $K$ a maximal compact subgroup of $G$. We shall denote the homogeneous space by $X=G / K$. The group $G$ acts on itself by left multiplications, and this action projects to the action on $X$. Let 0 be the fixed point of $K$ in $X$, then the natural projection

$$
\pi: G \rightarrow X
$$

is given by

$$
\pi: g \mapsto g(0)
$$

The Cartan decomposition corresponding to $K$ is

$$
\mathfrak{g}=\mathfrak{k} \oplus \mathfrak{p},
$$

where $\mathfrak{k}$ is the Lie algebra of $K, \operatorname{ad}(K) \mathfrak{p} \subset \mathfrak{p}$, and $\mathfrak{p}$ is the orthogonal complement to $\mathfrak{k}$ with respect to the Killing form on $\mathfrak{g}$. The differential $(d \pi)_{e}$ at the identity of $G$ identifies $\mathfrak{p}$ with $T_{0}(X)$. The kernel of $(d \pi)_{e}$ is $\mathfrak{k}($, 9 , p. 208).

To each $Y \in \mathfrak{g}$ is associated a left-invariant differential operator on $C^{\infty}(G)$ also denoted by $Y$,

$$
Y f(g)=\left.\frac{d}{d t} f(g \cdot \exp t Y)\right|_{t=0}
$$

this linear map from $\mathfrak{g}$ into the algebra $D(G)$ of left-invariant differential operators on $G$ extends to an isomorphism of $U(\mathfrak{g})$, the universal enveloping algebra of the Lie algebra $\mathfrak{g}$ (with complex coefficients) onto $D(G)$. In particular, one may consider differential operators $Y$ for $Y \in \mathfrak{g}^{c}$, the complexification of $\mathfrak{g}$. On $G^{0}$ the center $Z(\mathfrak{g})$ of $U(\mathfrak{g})$ corresponds to the left and right invariant operators and is isomorphic to a polynomial ring in $\ell$ letters where $\ell$ is the real rank of $G$. 
The general definition of automorphic forms in the sense of HarishChandra [10] and Borel [3] assumes only that $\Gamma$ is a discrete subgroup of $G$. In this paper we study cusp forms, which are automorphic forms with some additional hypotheses imposed, in case when $\Gamma$ is not cocompact, concerning the behavior close to certain boundary points. Naturally, if $\Gamma$ is cocompact, every automorphic form is a cusp form.

Let $\Gamma$ be a lattice in $G$, and $V$ be a finite-dimensional complex vector space. In what follows $G L(V)$ will act on $V$ on the right, and $\rho: K \rightarrow G L(V)$ be a fixed (anti-)representation of $K$ in $G L(V)$. Let $($,$) be a Hermitian \rho(K)$-invariant inner product on $V$ and | | the corresponding norm. We define the norm \|\|$_{p}(1 \leq p<\infty)$ on measurable $V$-valued $\Gamma$-invariant on the left functions on $G$ as usual by

$$
\|F\|_{p}=\left(\int_{\Gamma \backslash G}|F(g)|^{p} d g\right)^{\frac{1}{p}}<\infty,
$$

where $d g$ is the Haar measure on the group $G$, and the norm $\|F\|_{\infty}$ as the essential supremum of $|F|$ on $G$, and let $L^{p}(\Gamma \backslash G) \otimes V(1 \leq p \leq \infty)$ be the space of such $F$ for which $\|F\|_{p}<\infty$.

Definition 2. A vector-valued function $F: G \rightarrow V$ is called $Z(\mathfrak{g})$ finite if $Z(\mathfrak{g}) \cdot F$ is annihilated by an ideal $I$ of $Z(\mathfrak{g})$ of finite codimension.

Remark. If $I$ has codimension 1 , this means that $F$ is an eigenfunction of every operator in $Z(\mathfrak{g})$.

Definition 3. A smooth vector-valued function $F: G \rightarrow V$ is called a cusp form for $\Gamma$ if

(3.1) $F$ is $\Gamma$-invariant on the left and $K$-equivariant on the right, i.e. $F(\gamma g k)=F(g) \rho(k)$ for any $\gamma \in \Gamma, g \in G$ and $k \in K$;

(3.2) $F$ is $Z(\mathfrak{g})$-finite;

(3.3) $F \in L^{\infty}(\Gamma \backslash G) \otimes V$.

Remarks. 1. The condition (3.2) implies that $F \in C^{\infty}(G)$ [3], hence a function $F$ which satisfies (3.2) satisfies (3.3) if and only if $|F|$ is bounded on $G$.

2. If $\Gamma$ is cocompact, the condition (3.3) is automatic.

Automorphic forms may be defined using an automorphy factor, i.e. a smooth map $\mu: G \times X \rightarrow G L(V)$ that satisfies the 1-cocycle property, i.e.

$$
\mu\left(g_{1} g_{2}, x\right)=\mu\left(g_{1}, g_{2}(x)\right) \mu\left(g_{2}, x\right)
$$

for all $g_{1}, g_{2} \in G$ and $x \in X$. 
If a smooth function $f: X \rightarrow V$ satisfies the equation

$$
(f \mid \gamma)(x):=f(\gamma(x)) \mu(\gamma, x)=f(x),
$$

for any $\gamma \in \Gamma$ and $x \in X$, then its "lift" to $G, \tilde{f}: G \rightarrow V$ defined by

$$
\tilde{f}(g)=f(g(0)) \mu(g, 0),
$$

satisfies (3.1) with $\rho(k)=\mu(k, 0)$.

Definition 4. Let $\mu$ be an automorphy factor. A smooth function $f: X \rightarrow V$ is called a cusp form of type $\mu$ if its lift to the group $G$ defined by (6) is a cusp form of the Definition 3 with $\rho(k)=\mu(k, 0)$.

We denote the space of cusp forms of type $\mu$ (as well as the space of their lifts to $G)$ by $S_{\mu}(\Gamma)$.

The space $L^{2}(\Gamma \backslash G) \otimes V$ is a Hilbert space with the inner product

$$
\left(F_{1}, F_{2}\right)=\int_{\Gamma \backslash G}\left(F_{1}(g), F_{2}(g)\right) d g
$$

corresponding to the norm \|\|$_{2}$ introduced above. Using the Cartan decomposition of the group $G$ corresponding to (2),

$$
g=g_{x} k,
$$

where $k \in K$ and $g_{x}$ depends only on $x=g(0) \in X$, and the cocycle property of $\mu$ (田), we obtain for the lifts of automorphic forms $\tilde{f}_{1}(g)=$ $f_{1}(x) \mu(g, 0)$ and $\tilde{f}_{2}(g)=f_{2}(x) \mu(g, 0)$ with $x=g(0)$, an analogue of the Petersson inner product

$$
\left(f_{1}, f_{2}\right):=\left(\tilde{f}_{1}, \tilde{f}_{2}\right)=\int_{\Gamma \backslash G / K}\left(f_{1}(x) \mu\left(g_{x}, 0\right), f_{2}(x) \mu\left(g_{x}, 0\right)\right) d V,
$$

where $d V$ is a $G$-invariant volume form on the symmetric space $X$.

Since $\Gamma$ is a lattice, we have

$$
L^{\infty}(\Gamma \backslash G) \otimes V \subset L^{2}(\Gamma \backslash G) \otimes V \subset L^{1}(\Gamma \backslash G) \otimes V .
$$

Therefore the integral (9) converges for cusp forms; in fact, it converges if either $f_{1}$ or $f_{2}$ is a cusp form. Thus $S_{\mu}(\Gamma) \subset L^{1}(\Gamma \backslash G) \otimes V$. Notice that in general the converse inclusion is not true (e.g. for $G=S L(2, \mathbb{R}), \Gamma=$ $S L(2, \mathbb{Z})$ and Maass forms, a residual Eisenstein series has behavior like $y^{a}$, for $a<\frac{1}{2}$ in the cusp; it satisfies (3.1) and (3.2), is in $L^{1}(\Gamma \backslash G)$ but is not bounded), although for holomorphic forms on a symmetric bounded domain it is (see Remark 1 below).

Let $X$ be a symmetric bounded domain. If $f$ is a holomorphic function on $X$, the condition (3.2) is satisfied [3]. Let $\mathcal{H}_{\mu}^{p}(\Gamma \backslash G)$ be the subspace of $L^{p}(\Gamma \backslash G) \otimes V$ consisting of holomorphic functions on $X$ which satisfy (5). Then $S_{\mu}(\Gamma)=\mathcal{H}_{\mu}^{\infty}(\Gamma \backslash G)$. 
Remarks. 1. For holomorphic automorphic forms on a symmetric bounded domain $X$ all spaces $\mathcal{H}_{\mu}^{p}(\Gamma \backslash G)(1 \leq p \leq \infty)$ coincide for suitable $\mu$ bye Satake's theorem ([24], Exp. 9, Supp., [2], Ch. 11).

2. Although in general, $\operatorname{dim} S_{\mu}=\infty$, for holomorphic automorphic forms on a symmetric bounded domain $X, \operatorname{dim} S_{\mu}<\infty$ ([22], Ch. 4, |11], [2], Ch. 11).

Since $\Gamma$ is a lattice, it is finitely generated (see [5] for rank one and 17] for higher rank), and the homogeneous space $\Gamma \backslash G$ has a finite number of cusps (see |5] for rank one and [21], Ch. 8 for higher rank). The behavior of cusp forms in the cusps is studied by mapping each cusp to an unbounded Siegel domain by a partial Cayley transform and considering Fourier-Jacobi expansion of the transformed form. By Satake's theorem, cusp forms in $S_{\mu}(\Gamma)$ are characterized by the property that " $0{ }^{\text {th }}$ Fourier coefficient" of the transformed form on Sigel domain is equal to zero, hence they are rapidly decreasing at all cusps. We shall make use of this fact in $\$ 8.2$.

1.2. The relative Poincaré series. Let $\Gamma_{0}$ be a subgroup of $\Gamma$. The following construction allows to "extend" an automorphic form for $\Gamma_{0}$ to an automorphic form for $\Gamma$.

Theorem 5. Let $\Gamma_{0}$ be a subgroup of $\Gamma, f: X \rightarrow V$ be an automorphic form of type $\mu$ for $\Gamma_{0}$, and $\tilde{f}$ be its lift to the group $G$ by the formula (6) such that

1. $\tilde{f}$ is $Z(\mathfrak{g})$-finite,

2. $\tilde{f} \in L^{1}\left(\Gamma_{0} \backslash G\right) \otimes V$,

Then the series $\Theta_{\Gamma_{0}}=\sum_{\gamma \in \Gamma_{0} \backslash \Gamma} f \mid \gamma$, called the relative Poincaré series for $\Gamma_{0}$, converges absolutely and uniformly on compact sets, and represents a function satisfying (3.1), (3.2), and belonging to $L^{1}(\Gamma \backslash G) \otimes V$.

The proof follows the lines of the argument of Harish-Chandra for the Poincaré series (see [2] or [3]). It is proved that the series converges absolutely and uniformly on compact sets and satisfies (3.1) and (3.2). For cocompact $\Gamma$ this proves that $\Theta_{\Gamma_{0}}$ is a cusp form. It $\Gamma$ is a lattice, it follows that $\tilde{\Theta}_{\Gamma_{0}} \in L^{1}(\Gamma \backslash G) \otimes V$ which, without additional assumptions does not imply that $\Theta_{\Gamma_{0}}$ is a cusp form. However, for holomorphic automorphic forms on a symmetric bounded domain, according to Satake's theorem, the relative Poincaré series $\Theta_{\Gamma_{0}}$ are cusp forms.

If $X=G / K$ is a symmetric bounded domain, then any absolutely integrable function on $X$, e.g. any polynomial on $X$, produces a holomorphic cusp form for the trivial $\Gamma_{0}=\{e\}$ (see [2], Ch. 11).

If $\Gamma_{0}$ is a parabolic subgroup of $\Gamma$, the above construction gives socalled Poincaré-Eisenstein series. 
For general $\Gamma_{0}$, it is not easy to find a function $f$ which satisfies conditions 1 and 2 of Theorem 5. We shall give a construction for cyclic loxodromic subgroups of isometries of complex hyperbolic spaces in $\oint 6.3$.

\section{The HOMOGENEOUS FLOW}

2.1. Definitions. From now on we suppose, in addition to the standing assumptions of $\$ 1.1$, that $G$ is of real rank one and with finite center (and therefore non-compact simple). Then any non-zero $\mathfrak{D} \in \mathfrak{p}$ defines a maximal abelian subspace of $\mathfrak{p}, \mathfrak{a}=\mathbb{R} \mathfrak{D}$, consisting of real semisimple elements (cf. [9], pp. 401, 431). We fix a non-zero $\mathfrak{D} \in \mathfrak{p}$ and let

$$
A=\left\{\exp t \mathfrak{D}=a_{t} \mid t \in \mathbb{R}\right\}
$$

be the corresponding maximal split abelian subgroup of $G$. Acting by right multiplications on $G$, it defines a standard flow $\tilde{\alpha}_{t}$ on $G$,

$$
\tilde{\alpha}_{t}(g)=g a_{t} .
$$

An orbit of $g \in G,\left\{g a_{t} \mid t \in \mathbb{R}\right\}$ projects by (11) to a geodesic $\left\{g a_{t}(0)=g a_{t} g^{-1}\left(x_{g}\right) \mid t \in \mathbb{R}\right\}$ on $X$ passing through $x_{g}=g(0)$ ([9], p. 208). The subgroup $A$ itself projects on $X$ to the "standard geodesic"

$$
\mathfrak{I}=\left\{a_{t}(0) \mid t \in \mathbb{R}\right\}
$$

passing through 0 . There is a subgroup $W \subset K$ which, acting on $X$ on the left by isometries, fixes $\mathfrak{I}$ pointwise. Then $A W=W A=Z(A)$, the centralizer of $A$.

Definition 6. An element $g \in G$ is called hyperbolic (or regular) if it is conjugate to an element in $A$.

Definition 7. An element $g \in G$ is called loxodromic if it is conjugate to an element in $Z(A)$.

Any loxodromic (and, therefore, hyperbolic) element in $G$ fixes a geodesic in $X$, called its axis and has two fixed points in the boundary $\partial X$. It is easy to see that geodesics in $X$ are exactly the axes of loxodromic elements in $G$.

Geodesics naturally lift to $G / W$ which can be identified with the unit tangent bundle $S(G / K)$. Recall that as subgroups of $G$ acting on the space $X=G / K$ on the left by isometries, $K$ fixes the point $0 \in X$, and $W \subset K$ fixes $\mathfrak{I}$ pointwise, and hence its unit tangent vector. Thus we have two natural mappings:

$$
\pi: G \rightarrow G / K
$$


and

$$
\sigma: G \rightarrow G / W
$$

Defining the mapping

$$
\tau: G / W \rightarrow G / K
$$

such that $\tau \circ \sigma=\pi$ we see that $G / W$ is a sphere bundle over $G / K$ with the fiber at each point identified with $K / W$, the unit tangent space at this point. For any geodesic $C$ in $X$ passing through a point $x_{0}$ there exists a $g \in G$ mapping $\mathfrak{I}$ into $C$ in such a way that $g(0)=x_{0}$. This follows from the fact that the group $G$ acts transitively on the unit tangent bundle $S(X)=G / W$. The transformation $g$ is not unique but is determined up to the right multiplication by the subgroup $W$ which fixes every point of $\mathfrak{I}$. Thus we obtain a family of lifts of $C$, $\left\{g w a_{t} \mid w \in W, t \in \mathbb{R}\right\}$ to the group $G$ parametrized by the group $W$, which are orbits of the flow $\tilde{\alpha}_{t}$. Since the subgroups $A$ and $W$ commute, the flow $\tilde{\alpha}_{t}$ projects to the factor $G / W$ to the geodesic flow denoted by $\alpha_{t}$ :

$$
\alpha_{t}(\sigma(g))=\sigma\left(\tilde{\alpha}_{t}(g)\right) .
$$

We see that all lifts $\left\{g w a_{t} \mid w \in W, t \in \mathbb{R}\right\}$ project to the orbit $\alpha_{t}(g W)$ of the geodesic flow on $G / W$.

Let $\Gamma$ be a lattice in $G$ and $\Gamma \backslash X=M$. The action of $A$ descends to the factor $\Gamma \backslash G$ and defines a homogeneous flow $\tilde{\varphi}_{t}$ on $\Gamma \backslash G$ denoted by $\tilde{\varphi}_{t}$

$$
\tilde{\varphi}_{t}(\Gamma g)=\Gamma g a_{t}
$$

The mappings $\pi, \sigma$ and $\tau$ also descend to the corresponding left factors by $\Gamma$, and the homogeneous flow $\tilde{\varphi}_{t}$ projects to the geodesic flow on $S(M)=\Gamma \backslash G / W$, denoted by $\varphi_{t}$, by the formula:

$$
\varphi_{t}(\sigma(g))=\sigma\left(\tilde{\varphi}_{t}(g)\right) \text {. }
$$

For the base point $x=g(0)=\pi(g) \in X$ we shall use the same notation:

$$
\varphi_{t}(x)=\pi\left(\tilde{\varphi}_{t}(g)\right) \text {. }
$$

Respectively, we have two differential operators: on $C^{\infty}(\Gamma \backslash G)$,

$$
\mathfrak{D} f(g)=\left.\frac{d}{d t} f\left(\tilde{\varphi}_{t}(g)\right)\right|_{t=0},
$$

and on $C^{\infty}(\Gamma \backslash G / W)$,

$$
\mathcal{D} f(v)=\left.\frac{d}{d t} f\left(\varphi_{t}(v)\right)\right|_{t=0} .
$$

The following characterization of closed geodesics in $M=\Gamma \backslash X$ is immediate. Closed geodesics in $M$ are the axes of loxodromic elements 
in $\Gamma$. They are in one-to-finite correspondence with conjugacy classes of primitive loxodromic elements in $\Gamma$.

It is easy to see that closed geodesics in $M$ lift to closed orbits of the geodesic flow $\varphi_{t}$ on $\Gamma \backslash G / W$ while their lifts to $\Gamma \backslash G$ are not necessarily closed.

We shall denote a closed geodesic in $M$ corresponding to the axis of a loxodromic element $\gamma_{0} \in \Gamma$ in $\Gamma \backslash G / K$ as well as its lift to $\Gamma \backslash G / W$ by $\left[\gamma_{0}\right]$, and a family of lifts to $\Gamma \backslash G$ by $\left\{\left[\gamma_{0}\right]_{w} \mid w \in W\right\}$.

2.2. Dynamics of homogeneous and geodesic flows. It is a standard fact that there exists a left $G$-invariant Riemannian metric on $X=G / K$ relative to which $X$ is a symmetric space ( 19 , Ch.11, Th. 8.6). Since the real rank of $G$ is equal to one, this metric is of negative sectional curvature, which is the main reason of the following properties of the homogeneous flow $\tilde{\varphi}_{t}$ and the geodesic flow $\varphi_{t}$ which we review below.

A standard procedure ( $\mid 19 \|$, Ch. $11, \S 6)$ provides $G$-invariant Riemannian metrics on $G$ and $G / W$ (this can be done for any compact subgroup of $G$ in place of $W$ ) in such a way that the distance (which we will denote in both spaces by $d$ ) does not increase after projection: for any $g_{1}, g_{2} \in G$,

$$
d\left(\sigma\left(g_{1}\right), \sigma\left(g_{2}\right)\right) \leq d\left(g_{1}, g_{2}\right) .
$$

The Riemannian volume on $G$ coincides with the Haar measure.

We have seen that the homogeneous flow $\tilde{\varphi}_{t}$ on $\Gamma \backslash G$, considered as a fibered bundle over $\Gamma \backslash G / W$, projects to the geodesic flow $\varphi_{t}$ on the base $\Gamma \backslash G / W$. In addition, if $g_{1}$ and $g_{2}$ belong to the same fiber, i.e. $g_{2}=g_{1} w$ for some $w \in W$, we have

$$
d\left(\tilde{\varphi}_{t}\left(g_{1}\right), \tilde{\varphi}_{t}\left(g_{2}\right)\right)=d\left(g_{1}, g_{2}\right)
$$

by left-invariance of the metric and since $w$ and $a_{t}$ commute.

Let $D$ denote the differential of the flow. The geodesic flow $\varphi_{t}$ on $\Gamma \backslash G / W$ is Anosov (hyperbolic) ([16], Th. 17.6.2) with the corresponding $D \varphi_{t}$-invariant splitting of the tangent bundle of $\Gamma \backslash G / W$ :

$$
T(\Gamma \backslash G / W)=E^{0} \oplus E^{s} \oplus E^{u},
$$

and foliations $W^{0}$ (the orbit foliation), $W^{s}$, and $W^{u}$, respectively.

It follows that the homogeneous flow $\tilde{\varphi}_{t}$ is an isometric extension of $\varphi_{t}$ and, as such, is partially hyperbolic, i.e. there is a $C^{\infty} D \tilde{\varphi}_{t}$-invariant splitting of the tangent bundle of $\Gamma \backslash G$ :

$$
T(\Gamma \backslash G)=\tilde{E}^{0} \oplus \tilde{E}^{s} \oplus \tilde{E}^{u}
$$


with the following properties. The integral manifolds of the distribution $\tilde{E}^{0}$ form the neutral foliation denoted by $\tilde{W}^{0}$; its leaf through a point $g$ is $g W \times \tilde{\mathcal{O}}(g)$, where $\tilde{\mathcal{O}}(g)$ is the orbit of the flow, and is isometric in this case. The integral manifolds of the distributions $\tilde{E}^{s}\left(\tilde{E}^{u}\right)$ form the stable (unstable) foliation denoted by $\tilde{W}^{s}\left(\tilde{W}^{u}\right)$. Let us denote the distance along the leaves of the foliations $\tilde{W}^{s}$ and $\tilde{W}^{u}$ by $d^{s}$ and $d^{u}$, respectively. Then there exist $C, \lambda>0$ such that for any $g_{1}, g_{2}$ lying on the same leaf of $\tilde{W}^{j}(j=u, s)$,

$$
d^{j}\left(\tilde{\varphi}_{t}\left(g_{1}\right), \tilde{\varphi}_{t}\left(g_{2}\right)\right) \leq C e^{-\lambda|t|} d^{j}\left(g_{1}, g_{2}\right)
$$

for $j=s, t \geq 0$ and for $j=u, t \leq 0$.

The foliations $\tilde{W}^{0}, \tilde{W}^{s}, \tilde{W}^{u}$ are transversal and project to the corresponding foliations $W^{0}, W^{s}$, and $W^{u}$ of $\varphi_{t}$ with the same estimates (12).

The homogeneous flow $\tilde{\varphi}_{t}$ preserves the Haar measure on $\Gamma \backslash G$ while $\varphi_{t}$ preserves the Riemannian volume on $\Gamma \backslash G / W$.

\section{DyNAmical APPROACH TO CONSTRUCTION OF CUSP FORMS IN THE REAL RANK ONE CASE}

3.1. Cohomological equation for the homogeneous flow. The hyperbolic properties of the geodesic flow on $\Gamma \backslash G / W$ imply the Anosov Closing Lemma (see [16], Th. 6.4.15). As a consequence, the Livshitz Theorem holds:

The Livshitz Theorem. Let $f \in C^{\infty}(S(M))$ be such that it has zero integrals over all closed orbits of the geodesic flow on $S(M))$. Then there exists a Lipshitz function $F$ on $S(M)$ differentiable in the direction of the flow $\varphi_{t}$ (actually also $C^{\infty}(S(M))$ ), and such that

$$
\mathcal{D} F=f .
$$

It has been proved in 20 for Anosov flows on compact manifolds. The original proof works with minor alterationas for manifolds with cusps (the proof for Fuchsian groups with cusps are given in [13], Appendix).

By the Moore's ergodicity theorem ([25], Th. 2.2.6) the homogeneous flow on $\Gamma \backslash G$ is ergodic and hence topologically transitive, but it is not Anosov, the Closing Lemma does not hold, and hence a straightforward generalization of Livshitz Theorem does not hold either. However, the same conclusion holds under a stronger hypothesis, and the proof is similar to the proof of the Livshitz Theorem for the geodesic flow. 
Theorem 8 (Special Livshitz Theorem). Let $f \in C^{\infty}(\Gamma \backslash G)$ be such that for every closed geodesic $\left[\gamma_{0}\right]$ in $S(M)$ and every $w \in W$ the integral in $\Gamma \backslash G$

$$
\int_{\left[\gamma_{0}\right]_{w}} f\left(\tilde{\varphi}_{s}(g)\right) d s=0
$$

Then there exists a Lipschitz function $F$ on $\Gamma \backslash G$ constant on $W$-cosets and differentiable in the direction of the flow $\tilde{\varphi}_{t}$ and such that

$$
\mathfrak{D} F=f \text {. }
$$

Remark. We shall give a proof for real-valued functions. Then the theorem will obviously hold for functions valued in any $\mathbb{R}^{m}$.

Proof. The homogeneous flow on $\Gamma \backslash G$ is topologically transitive, i.e. there exists a $g \in G$ whose orbit $\tilde{\mathcal{O}}(g)=\left\{\tilde{\varphi}_{t}(g) \mid t \in \mathbb{R}\right\}$ is dense in $\Gamma \backslash G$. We define a function $F$ on this orbit by the formula

$$
F\left(\tilde{\varphi}_{t}(g)\right)=\int_{0}^{t} f\left(\tilde{\varphi}_{s}(g)\right) d s .
$$

We need to prove that $F$ satisfies a Lipschitz condition on $\tilde{\mathcal{O}}(g)$ and hence can be extended to $\Gamma \backslash G$ as a Lipschitz function. Given $\epsilon>0$, let $t_{1}<t_{2}$ be such that

$$
d\left(\tilde{\varphi}_{t_{1}}(g), \tilde{\varphi}_{t_{2}}(g)\right)<\epsilon
$$

We will show that

$$
\int_{t_{1}}^{t_{2}} f\left(\tilde{\varphi}_{s}(g)\right) d s=O(\epsilon)
$$

As it is customary, " $=O(x)$ " means that the expression is " $\leq C x$ " for some constant $C$. We will use this notation to avoid an accumulation of constants.

Since the homogeneous flow is not Anosov, we cannot hope to be able to approximate any $\epsilon$-close piece of the dense orbit by a closed one as for the geodesic flow. However, the close relation with the geodesic flow allows us to prove the following

Lemma 9 (Approximation Lemma). Using the notations above, there is a lift of a closed orbit of $\varphi_{t}$ to $\Gamma \backslash G,\left\{\tilde{\varphi}_{t}(\tilde{p}) \mid 0 \leq t \leq T\right\}$, such that for $0 \leq t \leq t_{2}-t_{1}$

$$
d\left(\tilde{\varphi}_{t_{1}+t}(g), \tilde{\varphi}_{t}(\tilde{p})\right)=O\left(\epsilon e^{-\lambda\left(\min \left(t, t_{2}-t_{1}-t\right)\right)}\right) .
$$

Proof. By (11) the projection of $\tilde{\mathcal{O}}(g)$ onto $\Gamma \backslash G / W$ is a dense orbit $\mathcal{O}(v)$ ) of the geodesic flow $\varphi_{t}$ with $v=\sigma(g)$, and it follows that

$$
d\left(\varphi_{t_{1}}(v), \varphi_{t_{2}}(v)\right)<\epsilon
$$




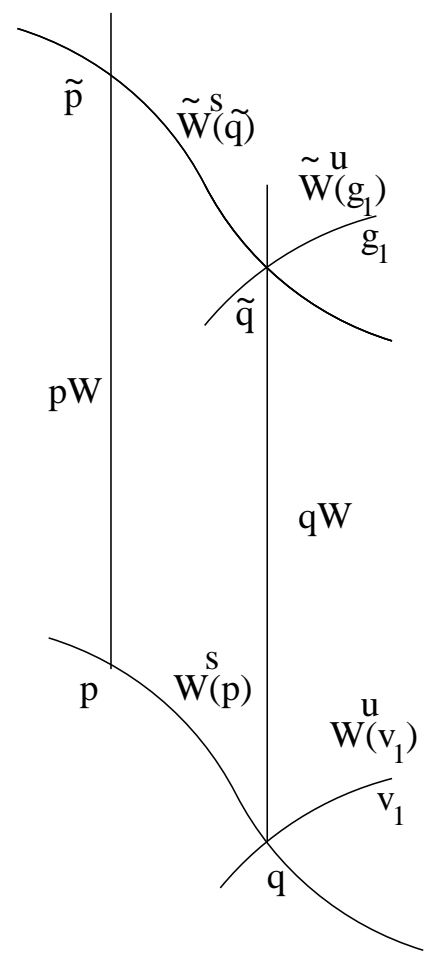

Figure 1.

Let $\left.\mathcal{O}(v)\right|_{t_{1}} ^{t_{2}}=\left\{\varphi_{t}(v) \mid t_{1} \leq t \leq t_{2}\right\}$ and $\left.\tilde{\mathcal{O}}(g)\right|_{t_{1}} ^{t_{2}}=\left\{\tilde{\varphi}_{t}(g) \mid t_{1} \leq t \leq\right.$ $\left.t_{2}\right\}$ be the corresponding pieces of orbits. Let us denote $\varphi_{t_{1}} v=v_{1}$ $\tilde{\varphi}_{t_{1}} g=g_{1}, \varphi_{t_{2}} v=v_{2}$ and $\tilde{\varphi}_{t_{2}} g=g_{2}$. Since the geodesic flow $\varphi_{t}$ is Anosov, by Anosov Closing Lemma there exists a $p \in \Gamma \backslash G / W$ such that $d\left(p, v_{1}\right)=O(\epsilon)$ and its orbit $\mathcal{O}(p)=\left\{\varphi_{t}(p) \mid t \in \mathbb{R}\right\}$ is closed, i.e. $\varphi_{T}(p)=p$ with the period $T$ satisfying $\left|T-\left(t_{2}-t_{1}\right)\right|=O(\epsilon)$. The point $p$ can be chosen such that the leaf of the unstable foliation containing $v_{1}, W^{u}\left(v_{1}\right)$, and a leaf of the stable foliation containing $p$, $W^{s}(p)$ intersect, and since they are transversal, they intersect in a point $W^{s}(p) \cap W^{u}\left(v_{1}\right)=q$, and $d^{u}\left(v_{1}, q\right)=O(\epsilon)$.

Lift $W^{u}\left(v_{1}\right)$ to $\tilde{W}^{u}\left(g_{1}\right)$. Since $q \in W^{u}\left(v_{1}\right)$ its lift $\tilde{W}^{u}\left(g_{1}\right)$ intersects $q W$, so let $\tilde{W}^{u}\left(g_{1}\right) \cap q W=\tilde{q}$, and similarly, $\tilde{W}^{s}(\tilde{q})$ intersects $p W$, $\tilde{W}^{s}(\tilde{q}) \cap p W=\tilde{p}$, and $\tilde{\varphi}_{T}(\tilde{p}) \in p W$ (see Fig. 1 which represents the picture in the direction transversal to the orbits).

Since the distance between $\varphi_{t}(p)$ and $\varphi_{t}(q)$ decreases exponentially for $t>0$, and $d^{s}(p, q)=O(\epsilon)$, we have

$$
d^{s}\left(\varphi_{t}(p), \varphi_{t}(q)\right)=O\left(\epsilon e^{-\lambda t}\right)
$$


and since all leaves are transversal we have the same estimate on $\tilde{W}^{s}(\tilde{q})$;

$$
d^{s}\left(\tilde{\varphi}_{t}(\tilde{p}), \tilde{\varphi}_{t}(\tilde{q})\right)=O\left(\epsilon e^{-\lambda t}\right) .
$$

Similarly, since the distance between $\varphi_{t_{2}-t_{1}-t}\left(v_{1}\right)$ and $\varphi_{t_{2}-t_{1}-t}(q)$ decreases exponentially for $t>0$, and $d^{u}\left(\varphi_{t_{2}-t_{1}}\left(v_{1}\right), \varphi_{t_{2}-t_{1}}(q)\right)=O(\epsilon)$, we have

$$
d^{u}\left(\varphi_{t}\left(v_{1}\right), \varphi_{t}(q)\right)=O\left(\epsilon e^{-\lambda\left(t_{2}-t_{1}-t\right)}\right)
$$

and the same estimate on $\tilde{W}^{s}(\tilde{q})$ :

$$
d^{u}\left(\tilde{\varphi}_{t}\left(g_{1}\right), \tilde{\varphi}_{t}(\tilde{q})\right)=O\left(\epsilon e^{-\lambda\left(t_{2}-t_{1}-t\right)}\right) .
$$

Therefore, we have simultaneous estimates

$$
d\left(\varphi_{t_{1}+t}(v), \varphi_{t}(p)\right)=O\left(\epsilon e^{-\lambda\left(\min \left(t, t_{2}-t_{1}-t\right)\right)}\right),
$$

and

$$
d\left(\tilde{\varphi}_{t_{1}+t}(g), \tilde{\varphi}_{t}(\tilde{p})\right)=O\left(\epsilon e^{-\lambda\left(\min \left(t, t_{2}-t_{1}-t\right)\right)}\right) .
$$

$0 \leq t \leq t_{2}-t_{1}$

It follows from (16) that

$$
\mid \int_{t_{1}}^{t_{2}} f\left(\tilde{\varphi}_{s}(g)\right) d s-\int_{0}^{T} f\left(\tilde{\varphi}_{s}(\tilde{p}) d s \mid=O(\epsilon)\right.
$$

and since by the hypothesis

$$
\int_{0}^{T} f\left(\tilde{\varphi}_{s}(\tilde{p}) d s=0\right.
$$

we obtain the required estimate (15). This proves the claim. Thus $F$ can be extended from the dense orbit to a Lipschitz function in $\Gamma \backslash G$. Since $\mathfrak{D} F=f$ on the dense orbit, it follows that $F$ is differentiable in the direction of the homogeneous flow and $\mathfrak{D} F=f$ in $\Gamma \backslash G$.

A similar argument shows that the function $F$ is constant on $W$ cosets. For, let $\sigma\left(g_{1}\right)=\sigma\left(g_{2}\right)=v$. There are $g_{1}^{\prime}$ and $g_{2}^{\prime}$ on the dense orbit of $\tilde{\varphi}_{t} \epsilon$-close to $g_{1}$ and $g_{2}$ respectively. Then the projection to $\Gamma \backslash G / W$ is an $\epsilon$-close orbit of $\varphi_{t}$. Find a closed orbit of $\varphi_{t} \epsilon$-close to the projection, and lift it back to $\Gamma \backslash G$ in the same manner we described before. It follows from the exponential estimates that

$$
\left|F\left(g_{2}^{\prime}\right)-F\left(g_{1}^{\prime}\right)\right|=O(\epsilon),
$$

and as $\epsilon \rightarrow 0$, we obtain $F\left(g_{1}\right)=F\left(g_{2}\right)$. 


\subsection{Spanning of cusp forms via relative Poincaré series in the} real rank one case. Our program of constructing spanning sets for $S_{\mu}(\Gamma)$ consists of three steps.

(1) Construction of relative Poincaré series associated to closed geodesics in $M=\Gamma \backslash G / K$. Fix a finite-dimensional complex vector space $V$ and an automorphy factor $\mu: G \times X \rightarrow G L(V)$. For each loxodromic element $\gamma_{0} \in \Gamma$ find a function $q_{\gamma_{0}}: X \rightarrow V$ which satisfied conditions of Theorem 5 for the subgroup $\Gamma_{0}=\left\langle\gamma_{0}\right\rangle$. Having such a function, we could apply Theorem 5 to obtain a relative Poincaré series $\Theta_{\gamma_{0}}$, and prove that they are cusp forms.

(2) The Period formula. Let $f \in S_{\mu}(G), \gamma_{0} \in \Gamma$ loxodromic, and $\tilde{f}$ be the lift of $f$ to $G$. Then for any lift $\left[\gamma_{0}\right]_{w}, w \in W$ of closed geodesic $\left[\gamma_{0}\right]$ to $G$,

$$
\left(f, \Theta_{\gamma_{0}}\right)=C \int_{\left[\gamma_{0}\right]_{w}} \tilde{f} d t
$$

with an explicit constant $C$ depending only on $\left[\gamma_{0}\right]_{w}$.

(3) Cohomological equation for cusp forms. In order to prove that the relative Poincare series $\left\{\Theta_{\gamma_{0}}, \gamma_{0} \in \Gamma\right.$ loxodromic $\}$ span $S_{\mu}(\Gamma)$, we assume that there is a cusp form $f \in S_{\mu}(G)$ such that $\left(f, \Theta_{\gamma_{0}}\right)=0$ for all relative Poincaré series $\Theta_{\gamma_{0}}$. Then by (2), $\tilde{f}$ satisfies the Special Livshitz Theorem (Theorem 8), and we obtain a smooth solution $F$ of the cohomological equation $\mathfrak{D} F=\tilde{f}$, which means that $\tilde{f}$ is a coboundary. Proving that cusp forms cannot be coboundaries will imply the result.

We are able to carry out this program in the case when $K$ has nontrivial 1-dimensional representations. In this case the symmetric space is Hermitian of real rank one, and by the classification of symmetric spaces ([9], p. 518), a complex hyperbolic space

$$
\mathbb{H}_{\mathbb{C}}^{n}=S U(n, 1) / S(U(n) \times U(1))
$$

for some $n \geq 1$. The rest of the paper is devoted to this case.

\section{The COMPleX HyPerbolic SPACE}

4.1. The unit ball model. The group $G=S U(n, 1)$ is the group of $(n+1) \times(n+1)$ complex unimodular matrices preserving the Hermitian form

$$
\langle z, w\rangle=z_{1} \bar{w}_{1}+\cdots+z_{n} \bar{w}_{n}-z_{n+1} \bar{w}_{n+1}
$$


on $\mathbb{C}^{n, 1}$. In other words,

$$
\begin{gathered}
G=\left\{A \in S L(n+1, \mathbb{C}) \mid\langle A \cdot z, A \cdot w\rangle=\langle z, w\rangle \forall z, w \in \mathbb{C}^{n, 1}\right\} \\
=\left\{A \in S L(n+1, \mathbb{C}) \mid A^{T} \cdot S \cdot \bar{A}=S\right\},
\end{gathered}
$$

where $1_{n}$ denotes the $n \times n$ identity matrix, $S=\left(\begin{array}{cc}1_{n} & 0 \\ 0 & -1\end{array}\right)$ and $\cdot$ is used for matrix multiplication.

The maximal compact subgroup of $G$

$$
K=S(U(n) \times U(1))=\left\{\left(\begin{array}{cc}
u_{n} & 0 \\
0 & a
\end{array}\right) \mid u_{n} \in U(n), a=\left(\operatorname{det} u_{n}\right)^{-1}\right\} .
$$

The symmetric space $G / K$ is called the complex hyperbolic space and is denoted by $\mathbb{H}_{\mathbb{C}}^{n}$. It can be identified with the projectivised space of negative vectors $z \in \mathbb{C}^{n, 1}$, i.e. such that $\langle z, z\rangle<0$ :

$$
\mathbb{H}_{\mathbb{C}}^{n} \cong \mathbb{P}\left(\left\{z \in \mathbb{C}^{n, 1} \mid\langle z, z\rangle<0\right\}\right),
$$

or, equivalently, with the set of negative lines in $\mathbb{C}^{n, 1}$, or in homogeneous coordinates, with the unit ball in $\mathbb{C}^{n}$ :

$$
B^{n}=\left\{z \in \mathbb{C}^{n} \mid z_{1} \bar{z}_{1}+\cdots+z_{n} \bar{z}_{n}<1\right\} .
$$

The last identification is obtained by the biholomorphic embedding

$$
\begin{gathered}
\mathbb{C}^{n} \rightarrow \mathbb{P}\left(\mathbb{C}^{n, 1}\right) \\
z \mapsto\left(\begin{array}{l}
z \\
1
\end{array}\right) .
\end{gathered}
$$

of $\mathbb{C}^{n}$ onto the affine chart of $\mathbb{P}\left(\mathbb{C}^{n, 1}\right)$ defined by $z_{n+1} \neq 0 . B^{n}$ is a bounded domain in $\mathbb{C}^{n}$ and hence a complex manifold. An invariant Riemannian metric on $B^{n}$ of the form $d s^{2}=g_{i j} d z_{i} d \overline{z_{j}}$ obtained by a standard construction $(\llbracket 19]$, Ch. $9, \S 6)$ is of negative sectional curvature and is called the Bergman metric. We normalize it according to [7], (III.1.3) so that the sectional curvature is pinched between -1 and $-\frac{1}{4}$. Using [9], Ch. 8, Prop. 2.5 one easily obtains the following relation between the volume form $d V$ on $B^{n}$ corresponding to the Riemannian metric and the Euclidean volume form $d V_{E}$ on $\mathbb{C}^{n}$ :

$$
d V=\frac{4^{n} d V_{E}}{(-\langle z, z\rangle)^{n+1}} \text {. }
$$

Using the above terminology, we call a vector $z \in \mathbb{C}^{n, 1}$ positive if $\langle z, z\rangle>0$ and a null vector if $\langle z, z\rangle=0$. We shall always assume that negative and null vectors are represented in the form (17), and keep the notation $\langle z, w\rangle=z_{1} \bar{w}_{1}+\cdots+z_{n} \bar{w}_{n}-1$ for them. 
4.2. The tangent bundle. For each point $z=\left(z_{1}, \ldots, z_{n}\right) \in B^{n}$ we write $z_{j}=x_{j}+i y_{j}$. The real tangent space at $z, T_{z}\left(B^{n}\right)=T_{z}$ refers to the tangent space of the underlying $2 n$-dimensional real $C^{\infty}$ manifold and has a basis

$$
\frac{\partial}{\partial x_{1}}(z), \frac{\partial}{\partial y_{1}}(z), \ldots, \frac{\partial}{\partial x_{n}}(z), \frac{\partial}{\partial y_{n}}(z)
$$

The vector fields $\frac{\partial}{\partial z_{j}}=\frac{1}{2}\left(\frac{\partial}{\partial x_{j}}-i \frac{\partial}{\partial y_{j}}\right)$ and $\frac{\partial}{\partial \bar{z}_{j}}=\frac{1}{2}\left(\frac{\partial}{\partial x_{j}}+i \frac{\partial}{\partial y_{j}}\right)$ form a basis of the complexification $T_{z}^{c}$ of $T_{z}$. The real tangent space $T_{z}$ can be identified with the $n$-dimensional complex subspace of $T_{z}^{c}$ of the vectors in the form $\eta=\sum_{j=1}^{n} \eta_{j} \frac{\partial}{\partial z_{j}}+\bar{\eta}_{j} \frac{\partial}{\partial \bar{z}_{j}}$, where $\eta_{j} \in \mathbb{C}$. We shall always refer to the tangent vectors being in the form $\eta=\left(\begin{array}{l}\eta_{1} \\ \ldots \\ \eta_{n}\end{array}\right)\left(\eta_{j} \in \mathbb{C}\right)$.

4.3. The action of $S U(n, 1)$. The group $G=S U(n, 1)$ acts on $B^{n}$ by biholomorphic transformations (automorphisms): for

$$
\begin{gathered}
g=\left(\begin{array}{cccc}
a_{11} & \ldots & a_{1 n} & b_{1} \\
\cdots & \ldots & \ldots & \ldots \\
a_{n 1} & \ldots & a_{n n} & b_{n} \\
c_{1} & \ldots & c_{n} & d
\end{array}\right), \\
g(z)=\left(\frac{a_{11} z_{1}+\cdots+a_{1 n} z_{n}+b_{1}}{c_{1} z_{1}+\cdots+c_{n} z_{n}+d}, \ldots, \frac{a_{n 1} z_{1}+\cdots+a_{n n} z_{n}+b_{n}}{c_{1} z_{1}+\cdots+c_{n} z_{n}+d}\right)
\end{gathered}
$$

(sometimes we will also use $g z$ for this action), which are isometries of $B^{n}$ with respect to the Bergman metric. This action corresponds to the left multiplication on $G$. The differential of $g: B^{n} \rightarrow B^{n}$ at $z$,

$$
g_{*}: T_{z} \rightarrow T_{g(z)}
$$

is given by the Jacobian matrix which can be written as

$$
J(g, z)=(c \cdot z+d)^{-2}(A(c \cdot z+d)-(A \cdot z+b) \cdot c),
$$

where $A=\left(\begin{array}{ccc}a_{11} & \ldots & a_{1 n} \\ \ldots & \ldots & \ldots \\ a_{n 1} & \ldots & a_{n n}\end{array}\right), b=\left(\begin{array}{c}b_{1} \\ \ldots \\ b_{n}\end{array}\right), c=\left(\begin{array}{lll}c_{1} & \ldots & c_{2}\end{array}\right), z=\left(\begin{array}{c}z_{1} \\ \ldots \\ z_{n}\end{array}\right)$.

A direct calculation shows that

$$
\operatorname{det} J(g, z)=(c \cdot z+d)^{-(n+1)}=\left(c_{1} z_{1}+\cdots+c_{n} z_{n}+d\right)^{-(n+1)} .
$$

The group of biholomorphic automorphisms of $B^{n}$ is actually

$$
P U(n, 1)=G / Z
$$

where

$$
Z=\left\{1_{n+1}, \epsilon 1_{n+1}, \ldots, \epsilon^{n} 1_{n+1}\right\}
$$


is the center of $G$ ( $\epsilon$ is the primitive $(n+1)^{t h}$ root of 1$)$.

4.4. The Cartan decomposition. The Lie algebra of the group $G$ is $\mathfrak{g}=\mathfrak{s u}(n, 1)$. The Cartan decomposition (2) is

$$
\mathfrak{g}=\mathfrak{k} \oplus \mathfrak{p} .
$$

$\mathfrak{k}$ is the Lie algebra of $K$ consisting of $(n+1) \times(n+1)$ complex matrices of the form

$$
\left(\begin{array}{cc}
U & 0 \\
0 & i \lambda
\end{array}\right)
$$

where $U$ is an $n \times n$ skew Hermitian matrix, $\lambda \in \mathbb{R}$, and $\operatorname{tr} U+i \lambda=0$, and $\mathfrak{p}$ consists of matrices

$$
\left(\begin{array}{cc}
0_{n} & \eta \\
{ }^{\bar{\eta}} & 0
\end{array}\right)
$$

where $\eta=\left(\begin{array}{l}\eta_{1} \\ \cdots \\ \eta_{n}\end{array}\right) \in \mathbb{C}^{n}$.

The differential at the identity $(d \pi)_{e}$ of the natural mapping

$$
\begin{aligned}
& \pi: G \rightarrow B^{n} \\
& \pi: g \mapsto g(0) .
\end{aligned}
$$

$\operatorname{maps}\left(\begin{array}{ll}0_{n} & \eta \\ { }^{t} \bar{\eta} & 0\end{array}\right) \in \mathfrak{p}$ to the tangent vector $\eta=\left(\begin{array}{l}\eta_{1} \\ \ldots \\ \eta_{n}\end{array}\right) \in T_{g(0)}$ thus identifying $\mathfrak{p}=T_{0}(G / K)$ with $T_{g(0)}\left(B^{n}\right)$.

Any element

$$
g=\left(\begin{array}{cccc}
a_{11} & \ldots & a_{1 n} & b_{1} \\
\ldots & \ldots & \ldots & \ldots \\
a_{n 1} & \ldots & a_{n n} & b_{n} \\
c_{1} & \ldots & c_{n} & d
\end{array}\right) \in G
$$

can be decomposed correspondingly according to (8) as follows:

$$
g=g_{z} \cdot k \text {. }
$$

The matrix $k \in K$ in the form $k=\left(\begin{array}{cc}u_{n} & 0 \\ 0 & e^{-i \psi}\end{array}\right)$, where $u_{n} \in U(n)$ and det $u_{n}=e^{i \psi}$, the matrix $g_{z}$ is hyperbolic whose entries depend only on $z=g(0)$, and represents the projection on the symmetric space $G / K$ 
(see [7], III.2.7), and

$$
d=\frac{e^{-i \psi}}{\sqrt{-\langle z, z\rangle}}
$$

4.5. The complex structure. Recall (匹19, Ch. 9) that the canonical complex structure on $B^{n}$ is a tensor field $J: z \rightarrow J_{z}$, where for each $z \in B^{n}, J_{z}$ is an automorphism of the tangent space $T_{z}$ such that $J_{z}^{2}=-I$ ( $I$ denotes the identity map of $\left.T_{z}\right)$. In local coordinates it is given by

$$
J_{z}\left(\frac{\partial}{\partial x_{j}}(z)\right)=\frac{\partial}{\partial y_{j}}(z), J_{z}\left(\frac{\partial}{\partial y_{j}}(z)\right)=-\frac{\partial}{\partial x_{j}}(z) .
$$

Restricting $J$ to $T_{0}\left(B^{n}\right)=\mathfrak{p}$ we obtain an automorphism $J_{0}: \mathfrak{p} \rightarrow \mathfrak{p}$ such that $J_{0}^{2} X=-X$ for all $X \in \mathfrak{p}$. Since $G$ is semisimple, there exists an element $Z_{0} \in Z(\mathfrak{k})$ such that $J_{0}=\operatorname{ad}_{\mathfrak{p}}\left(Z_{0}\right)$ and $\mathfrak{k}=\{X \in \mathfrak{g} \mid$ $\left.\left[Z_{0}, X\right]=0\right\}$ (19 , Ch. 11, Th. 9.6). We have

$$
Z_{0}=\left(\begin{array}{cc}
\frac{i}{n+1} 1_{n} & 0 \\
0 & -\frac{n i}{n+1}
\end{array}\right)
$$

and $J_{0}\left(\left(\begin{array}{cc}0_{n} & \eta \\ t \bar{\eta} & 0\end{array}\right)\right)=\left(\begin{array}{cc}0_{n} & i \eta \\ -i^{t} \bar{\eta} & 0\end{array}\right)$ for all $\left(\begin{array}{cc}0_{n} & \eta \\ { }^{t} \bar{\eta} & 0\end{array}\right) \in \mathfrak{p}$.

For each $z \in B^{n}$ the automorphism $J_{z}$ can be extended uniquely to a complex linear mapping of the complexification $T_{z}^{c}$ of $T_{z}$ onto itself also denoted by $J_{z}$, and satisfying $\left(J_{z}\right)^{2}=-I$. The eigenvalues of $J_{z}$ are therefore $i$ and $-i$. For $z=0$ we have $T_{0}^{c}\left(B^{n}\right)=\mathfrak{p}^{c}$ and the corresponding eigenspaces are

$$
\mathfrak{p}^{+}=\left\{Z \in \mathfrak{p}^{c} \mid J(Z)=i Z\right\} \quad \text { and } \quad \mathfrak{p}^{-}=\left\{Z \in \mathfrak{p}^{c} \mid J(Z)=-i Z\right\} .
$$

Then

$$
\mathfrak{p}^{+}=\{X-i J(X) \mid X \in \mathfrak{p}\}, \quad \mathfrak{p}^{-}=\{X+i J(X) \mid X \in \mathfrak{p}\} .
$$

and $\mathfrak{p}^{c}$ can be written as a direct sum of complex vector spaces

$$
\mathfrak{p}^{c}=\mathfrak{p}^{+} \oplus \mathfrak{p}^{-}
$$

Thus by (26) and (23)

$$
\mathfrak{p}^{+}=\left\{\left(\begin{array}{cc}
0_{n} & \eta \\
0 & 0
\end{array}\right) \mid \eta \in \mathbb{C}^{n}\right\}
$$

and

$$
\mathfrak{p}^{-}=\left\{\left(\begin{array}{ll}
0_{n} & 0 \\
{ }^{t} \eta & 0
\end{array}\right) \mid \eta \in \mathbb{C}^{n}\right\}
$$


Any $g \in G$ can be also decomposed according to Harish-Chandra ([3], p. 688, [9], Ch. VIII, §7) as

$$
g=g_{+} \cdot g_{0} \cdot g_{-},
$$

where $g^{+}=\exp p^{+}, p^{+} \in \mathfrak{p}^{+}, g^{-}=\exp p^{-}, p^{-} \in \mathfrak{p}^{-}$, and $g_{0} \in K^{c}$, the complexification of $K$. For $g=\left(\begin{array}{cccc}a_{11} & \ldots & a_{1 n} & b_{1} \\ \ldots & \ldots & \ldots & \ldots \\ a_{n 1} & \ldots & a_{n n} & b_{n} \\ c_{1} & \ldots & c_{n} & d\end{array}\right)$ we obtain

$$
g^{+}=\left(\begin{array}{cc}
1_{n} & z \\
0 & 1
\end{array}\right) \quad \text { and } \quad g^{-}=\left(\begin{array}{cc}
1_{n} & 0 \\
t_{w} & 1
\end{array}\right)
$$

where $z=\left(\begin{array}{c}\frac{b_{1}}{d} \\ \ldots \\ \frac{b_{n}}{d}\end{array}\right)$ and $w=\left(\begin{array}{c}\frac{c_{1}}{d} \\ \ldots \\ \frac{c_{n}}{d}\end{array}\right)$. Since $z=g(0)$ we see that the Harish-Chandra map

$$
\chi: G \rightarrow \mathfrak{p}^{+}
$$

given by

$$
g \mapsto \log g^{+}
$$

is exactly the map $\pi$ of (24) and the bounded domain $\chi(G) \subset \mathfrak{p}^{+}$is the unit ball $B^{n} \subset \mathbb{C}^{n}$.

Remark. Notice that in $(27) w \neq \bar{z}$.

The Bergman metric on $B^{n}$ introduced in $\$ 4.1$ is Hermitian, i.e. invariant by the complex structure $J$, and thus defines a Hermitian inner product on each tangent space $T_{z}\left(B^{n}\right)$.

\section{THE FRAME FLOW}

5.1. Geometric interpretation. Let $\Gamma$ be a lattice in $G$ and $\mathfrak{D}=$ $\left(\begin{array}{ccc}0_{n-1} & 0 & 0 \\ 0 & 0 & 1 \\ 0 & 1 & 0\end{array}\right) \in \mathfrak{p}$. The corresponding maximal split abelian subgroup of $G$

$$
A=\left\{a_{t}=\left(\begin{array}{ccc}
1_{n-1} & 0 & 0 \\
0 & \cosh t & \sinh t \\
0 & \sinh t & \cosh t
\end{array}\right) \mid t \in \mathbb{R}\right\}
$$


projects to the "standard geodesic"

$$
\mathfrak{I}=\left\{a_{t}(0)=\left(\begin{array}{c}
0 \\
\cdots \\
0 \\
\tanh t \\
1
\end{array}\right) \mid t \in \mathbb{R}\right\} .
$$

The subgroup of $K$ which commutes with $A$ is

$$
W=\left\{\left(\begin{array}{ccc}
u_{n-1} & 0 & 0 \\
0 & e^{-i \psi} & 0 \\
0 & 0 & e^{-i \psi}
\end{array}\right), \psi \in \mathbb{R} / 2 \pi \mathbb{Z}, \operatorname{det} u_{n-1}=e^{2 i \psi}\right\},
$$

As has been explained in $\$ 2.1$, acting by right multiplications on $G$, $A$ defines a homogeneous flow $\tilde{\varphi}_{t}$ on $\Gamma \backslash G$, which is called the frame flow in this case. The terminology comes from the fact that the group $P U(n, 1)$ may be identified with a principal $U(n)$-bundle over $B^{n}$. A fiber at the point $z \in B^{n}$ geometrically represents the space of $n^{-}$ frames: unitary frames of tangent vectors at $z$ with respect to the Hermitian inner product. Geometrically, the flow $\tilde{\varphi}_{t}$ acts as follows: along the geodesic it leaves invariant it acts by hyperbolic isometries whose differentials move the last vector along the geodesic at constant speed (this represents the projection of $\tilde{\varphi}_{t}$ to the geodesic flow $\varphi_{t}$ on $\Gamma \backslash G / W)$, and the remaining vectors of the frame by parallel translation ([19], Ch. 9, Th. 3.2). Notice that the distance with respect to the Bergman metric between two points differs from the parameter $t$ of the geodesic flow on the corresponding geodesic by the factor of 2 : $d\left(z, \varphi_{t}(z)\right)=2 t$.

5.2. Loxodromic elements in $S U(n, 1)$. As has been explained in the general setup in $\$ 2.1$, a loxodromic element $\gamma_{0}$ has the axis in $B^{n}$, and two fixed points in the boundary $\partial B^{n}$, one attracting, and one repelling. The fixed points correspond to the null eigenvectors of $\gamma_{0}$ with eigenvalues $\lambda$ and $\bar{\lambda}^{-1}$, and the remaining $n-1$ eigenvectors are positive with eigenvalues of absolute value 1 .

As we have pointed out earlier, the automorphisms of $B^{n}$ are in fact elements of $P U(n, 1)$. Therefore each loxodromic automorphism may be also represented by an element of $U(n, 1)$ with a real eigenvalue not equal to 1. Sometimes it is possible to find such an element in $S U(n, 1)$. Eigenvectors (in $\mathbb{C}^{n+1}$ ) and hence fixed points (on the boundary $\partial B^{n}$ ) do not depend on the choice of a representative in $U(n, 1)$. The following proposition gives an explicit formula for the transformation $T$ conjugating a loxodromic element in $U(n, 1)$ to an "almost hyperbolic" 
element with a nice fundamental domain. The check that given formulae work is routine. The explicit formula for $T$ will be used in $\S[7$.

Proposition 10. Let $\gamma_{0}$ be a loxodromic element in $U(n, 1)$ with eigenvectors $V_{1}, \ldots, V_{n-1}, X, Y$ and corresponding eigenvalues $\tau_{1}, \ldots, \tau_{n-1}, \lambda, \lambda^{-1}$, $\left|\tau_{j}\right|=1, j=1, \ldots, n-1, \lambda \in \mathbb{R},|\lambda|>1$. Let $V_{j}, j=1, \ldots, n-1$, be normalized so that $\left\langle V_{j}, V_{j}\right\rangle=1$ and $V_{1}$ be chosen so that the matrix $T:=\left(\begin{array}{lllll}V_{1} & \ldots & V_{n-1} & \frac{X}{\langle X, Y\rangle}+\frac{Y}{2} & \frac{X}{\langle X, Y\rangle}-\frac{Y}{2}\end{array}\right)$ has real positive determinant. Let $F_{0}$ be the Dirichlet fundamental domain for $\left\langle\gamma_{0}\right\rangle$ centered at $T(0)$.

Then

1) $T \in S U(n, 1)$,

2) $\gamma:=T^{-1} \cdot \gamma_{0} \cdot T=\left(\begin{array}{ccccc}\tau_{1} & 0 & \ldots & \ldots & 0 \\ \ldots & \ldots & \ldots & \ldots & \ldots \\ 0 & \ldots & \tau_{n-1} & 0 & 0 \\ 0 & \ldots & 0 & \frac{\lambda^{2}+1}{2 \lambda} & \frac{\lambda^{2}-1}{2 \lambda} \\ 0 & \ldots & 0 & \frac{\lambda^{2}-1}{2 \lambda} & \frac{\lambda^{2}+1}{2 \lambda}\end{array}\right)$ has null eigenvectors $X_{0}=\left(\begin{array}{c}0 \\ \ldots \\ 0 \\ 1 \\ 1\end{array}\right)$ and $Y_{0}=\left(\begin{array}{c}0 \\ \ldots \\ 0 \\ -1 \\ 1\end{array}\right)$,

3) the fundamental domain $T^{-1}\left(F_{0}\right)$ of $\langle\gamma\rangle$ is bounded by two hypersurfaces:

$$
H_{1}=\left\{w:\left|\frac{\lambda^{2}-1}{2 \lambda} w_{n}-\frac{\lambda^{2}+1}{2 \lambda}\right|=1\right\}
$$

and

$$
H_{2}=\left\{w:\left|\frac{\lambda^{2}-1}{2 \lambda} w_{n}+\frac{\lambda^{2}+1}{2 \lambda}\right|=1\right\} .
$$

\section{Automorphic Forms on the COMPleX Hyperbolic SPACE}

6.1. Automorphy factor. For any $g \in G$, along with the Jacobian matrix $J(g, z)$ and its determinant, the function

$$
j(g, z)=(\operatorname{det} J(g, z))^{\frac{1}{n+1}}=\left(c_{1} z_{1}+\cdots+c_{n} z_{n}+d\right)^{-1},
$$

is a 1-cocycle. Taking $\mu(g, z)=j(g, z)^{(n+1) \kappa}$ for an integer $\kappa \geq 1$ as an automorphy factor, we see that for $k \in K, \mu(k, 0)=\rho(k)=e^{(n+1) \kappa \psi}$ a 1 -dimensional representation of $K$. The operator $f \mid \gamma$ defined in (5) is well-defined on $P U(n, 1)$. Thus we obtain a space of cusp forms of weight $(n+1) \kappa$ denoted classically by $S_{(n+1) \kappa}(\Gamma)$ (see e.g. [23]). 
Using the formula (25) we see that

$$
\mu(g, 0)=d^{-(n+1) \kappa}=\left(e^{i \psi} \sqrt{-\langle z, z\rangle}\right)^{(n+1) \kappa},
$$

and therefore the inner product is given by the formula

$$
\left(f_{1}, f_{2}\right)=\int_{\Gamma \backslash \mathbb{H}_{\mathbb{C}}^{n}} f_{1} \overline{f_{2}}(-\langle z, z\rangle)^{(n+1) \kappa} d V
$$

6.2. Lift to the group. It is convenient to choose the following local (partial) coordinates $(z, \eta, \zeta)$ on $S U(n, 1)$ :

$$
z(g)=g(0) \in B^{n}, \eta(g)=J(g, 0) \cdot \iota \in S_{z}\left(B^{n}\right),
$$

where $\iota$ is the unit tangent vector at 0 to the "standard geodesic" $\mathfrak{I}$, and

$$
\zeta(g)=j(g, 0)=d^{-1}=\sqrt{(-\langle z, z\rangle)} e^{i \psi} \neq 0 .
$$

It is easy to check that the left multiplication by $g^{\prime} \in G$ corresponds to the action on $z \in B^{n}$ by a biholomorphic transformation $g^{\prime}(z)$ (20), on $\eta \in S_{z}\left(B^{n}\right)$ by the Jacobian matrix $J\left(g^{\prime}, z\right)$ (21), and on $\zeta$ by $j\left(g^{\prime}, z\right)$ (29). The lift of the automorphic form $f \in S_{(n+1) \kappa}(\Gamma)$ to $G$ (ब6) has a nice expression in these coordinates:

$$
\tilde{f}(g)=f(z) \zeta^{(n+1) \kappa} .
$$

\subsection{Construction of relative Poincaré series associated to closed}

geodesics. The following construction associates a cusp form to each

loxodromic element $\gamma_{0}=\left(\begin{array}{cccc}a_{11} & \ldots & a_{1 n} & b_{1} \\ \ldots & \ldots & \ldots & \ldots \\ a_{n 1} & \ldots & a_{n n} & b_{n} \\ c_{1} & \ldots & c_{n} & d\end{array}\right) \in \Gamma$. As a loxodromic

automorphism, $\gamma_{0}$ has two fixed points on the boundary $\partial B^{n}, X$ and $Y:\langle X, X\rangle=0$ and $\langle Y, Y\rangle=0, X$ repelling and $Y$ attracting. Then $Q_{\gamma_{0}}(z)=\langle z, X\rangle\langle z, Y\rangle \neq 0$ on the closure of $B^{n}$ except for the points $X$ and $Y$, and transforms under $\gamma_{0}$ as follows:

$$
Q_{\gamma_{0}}\left(\gamma_{0} z\right)=j\left(\gamma_{0}, z\right)^{2} Q_{\gamma_{0}}(z)=\left(c_{1} z_{1}+\cdots+c_{n} z_{n}+d\right)^{-2} Q_{\gamma_{0}}(z) .
$$

The function $q(z)=\frac{1}{Q_{\gamma_{0}}^{(n+1) \kappa}(z)}$ is an automorphic form of type $\mu(g, z)=$ $j(g, z)^{2(n+1) \kappa}$ for the subgroup $\Gamma_{0}=\left\langle\gamma_{0}\right\rangle$, and it satisfies the condition 1 of Theorem 5 since it is holomorphic. The condition 2 is also satisfied. To see that we write using (18)

$$
\int_{\Gamma_{0} \backslash B^{n}}|q(z)|(-\langle z, z\rangle)^{(n+1) \kappa} d V=4^{n} \int_{F_{0}} \frac{(-\langle z, z\rangle)^{(n+1)(\kappa-1)}}{(\langle z, X\rangle\langle z, Y\rangle)^{(n+1) \kappa}} d V_{E}
$$

where $F_{0}$ is a Dirichlet fundamental domain for $\Gamma_{0}$. Since the denominator of the expression in the second integral is equal to zero only at 
$z=X$ and $z=Y$, the expression represents a continuous function on $F_{0}$ for any integer $\kappa \geq 1$. Since $F_{0}$ is a bounded domain, the integral is finite. Thus we produce a relative Poincaré series

$$
\Theta_{\gamma_{0}, \kappa}(z)=\sum_{\gamma \in \Gamma_{0} \backslash \Gamma}(q \mid \gamma)(z)
$$

of weight $2(n+1) \kappa$ for each $\kappa \geq 1$, which belongs to $L^{1}(\Gamma \backslash G)$, and, by Satake's theorem is a cusp form. If $\gamma_{1}$ is conjugate to $\gamma_{0}$ in $\Gamma$, then it is easy to see that $\Theta_{\gamma_{1}, \kappa}(z)=\Theta_{\gamma_{0}, \kappa}(z)$. If two primitive loxodromic elements $\gamma_{0}, \gamma_{1} \in \Gamma$ have the same axis, then $\gamma_{1}=\gamma_{0} w$, where $w$ belongs to a compact subgroup of $K$ conjugate to $W$. It follows from discreteness of $\Gamma$ that if $n \geq 2$ finitely many elements in $\Gamma$ may have the same axis). It is a consequence of the period formula (Corollary 13) that their relative Poincaré series coincide.

\section{THE PERIOD FORMULA}

Theorem 11. For any $f \in S_{2(n+1) \kappa}(\Gamma)$ and any loxodromic element $\gamma_{0} \in \Gamma$,

$$
\left(f, \Theta_{\gamma_{0}, \kappa}\right)=|\alpha|^{-2(n+1) \kappa} C \int_{z_{0}}^{\gamma_{0} z_{0}} f(z) Q_{\gamma_{0}, \kappa}(z)^{(n+1) \kappa} d t .
$$

Here $z_{0}$ is any point on the axis of $\gamma_{0}$, and the integration is over the axis of $\gamma_{0}$ in $B^{n}, t$ is the parameter of the geodesic flow, $\alpha=-\frac{\langle X, Y\rangle}{2}$, and $C=\frac{(2(n+1) \kappa-n-1) !}{(((n+1) \kappa-1) !)^{2}} \pi^{n} 2^{2(n+1)(1-\kappa)-1}$ is a constant.

Proof. Let

$$
I:=\left(f, \Theta_{\gamma_{0}, \kappa}\right) .
$$

Since the series (34) converges absolutely, we can interchange summation and integration and, using standard "Rankin's method" obtain

$$
\begin{aligned}
I & =\int_{F=\Gamma \backslash B^{n}} f(z) \sum_{\gamma \in \Gamma_{0} \backslash \Gamma} \overline{(q \mid \gamma)(z)}(-\langle z, z\rangle)^{2(n+1) \kappa} d V \\
& =\sum_{\gamma \in \Gamma_{0} \backslash \Gamma} \int_{F} f(z) \overline{(q \mid \gamma)(z)}(-\langle z, z\rangle)^{2(n+1) \kappa} d V \\
& =\sum_{\gamma \in \Gamma_{0} \backslash \Gamma} \int_{\gamma F} f(z) \overline{q(z)}(-\langle z, z\rangle)^{2(n+1) \kappa} d V \\
& =\int_{F_{0}} f(z) \frac{(-\langle z, z\rangle)^{2(n+1) \kappa}}{(\langle X, z\rangle\langle Y, z\rangle)^{(n+1) \kappa}} d V,
\end{aligned}
$$

where $F_{0}$ is a fundamental domain for $\left\langle\gamma_{0}\right\rangle$.

We now make change of variables $w=T^{-1} z$, where $T$ is as in Proposition 10, mapping the "standard geodesic" $\mathfrak{I}$ into the axis of $\gamma_{0}$. The following lemma is checked easily. 
Lemma 12. $\langle z, X\rangle\langle z, Y\rangle=\bar{\alpha} j(T, w)^{2}\left(1-w_{n}^{2}\right)$, where $\alpha=-\frac{\langle X, Y\rangle}{2}$.

According to (18) we have

$$
d V=(2 i)^{n} \frac{d z_{1} \wedge d \bar{z}_{1} \wedge \ldots \wedge d z_{n} \wedge d \bar{z}_{n}}{(-\langle z, z\rangle)^{(n+1)}} .
$$

Let us denote $d V_{i}=d w_{i} \wedge d \bar{w}_{i} \wedge \ldots \wedge d w_{n} \wedge d \bar{w}_{n}$. Then

$$
\begin{aligned}
I & =(2 i)^{n} \int_{F_{0}} f(z) \frac{(-\langle z, z\rangle)^{2(n+1) k}}{(\langle X, z\rangle\langle Y, z\rangle)^{(n+1) \kappa}} \frac{d z_{1} \wedge d \bar{z}_{1} \wedge \ldots \wedge d z_{n} \wedge d \bar{z}_{n}}{(-\langle z, z\rangle)^{(n+1)}} \\
& =(2 i)^{n} \int_{T^{-1} F_{0}} f(T w) \frac{(-\langle T w, T w\rangle)^{2(n+1) k}}{(\langle X, T w\rangle\langle Y, T w\rangle)^{(n+1) \kappa}} \frac{d w_{1} \wedge d \bar{w}_{1} \wedge \ldots \wedge d w_{n} \wedge d \bar{w}_{n}}{(-\langle w, w\rangle)^{(n+1)}} \\
& =(2 i)^{n} \alpha^{-(n+1) \kappa} \int_{T^{-1} F_{0}} f(T w) \frac{(-\langle w, w\rangle)^{2(n+1) \kappa}|j(T, w)|^{4(n+1) \kappa}}{\left(1-\bar{w}_{n}^{2}\right)^{(n+1) \kappa}\left(j(T, w)^{2(n+1) \kappa}\right)} \frac{d V_{1}}{(-\langle w, w\rangle)^{(n+1)}} \\
& =(2 i)^{n} \alpha^{-(n+1) k} \int_{T^{-1} F_{0}} f(T w) \frac{(-\langle w, w\rangle)^{2(n+1) \kappa-(n+1)} j(T, w)^{2(n+1) \kappa}}{\left(1-\bar{w}_{n}^{2}\right)^{(n+1) k}} d V_{1} .
\end{aligned}
$$

Further calculations can be divided onto three parts:

Step 1. Show that

$$
\begin{gathered}
I=-\frac{2 \pi i}{2(n+1) \kappa-(n+1)+1}(2 i)^{n} \alpha^{-(n+1) \kappa} \times \\
\int_{T^{-1} F_{0} \cap\left\{w: w_{1}=0\right\}} f(T w) \frac{(-\langle w, w\rangle)^{2(n+1) \kappa-(n+1)+1}}{\left(1-\bar{w}_{n}^{2}\right)^{(n+1) \kappa}}(j(T, w))^{2(n+1) \kappa} d V_{2} .
\end{gathered}
$$

Step 2. Show that

$$
\begin{aligned}
& I=2^{n} i \alpha^{-(n+1) \kappa} \frac{(2(n+1) \kappa-n-1) !}{(2(n+1) \kappa-2) !}(2 \pi)^{n-1} \times \\
& \quad \int_{T^{-1} F_{0} \cap\left\{w: w_{1}=\ldots=w_{n-1}=0\right\}} f(T w) \frac{(-\langle w, w\rangle)^{2(n+1) \kappa-2}}{\left(1-\bar{w}_{n}^{2}\right)^{(n+1) \kappa}}(j(T, w))^{2(n+1) \kappa} d V_{n} .
\end{aligned}
$$

Step 3. Show that

$$
I=C \int_{z_{0}}^{\gamma_{0} z_{0}} f(z)(\langle z, X\rangle\langle z, Y\rangle)^{(n+1) \kappa} d t .
$$

Step 1. $\gamma=T^{-1} \gamma_{0} T$ leaves invariant $\left\{w: w_{1}=0\right\}$. The fundamental domain $T^{-1} F_{0}$ for $\gamma$ over which we take the integral can be described as $D_{1}=T^{-1} F_{0} \cap\left\{w: w_{1}=0\right\}$ with the disc $\left\{w: w_{2}=\right.$ const, $\ldots, w_{n}=$ const $\}$ of radius $\sqrt{1-w_{2} \bar{w}_{2}-\ldots-w_{n} \bar{w}_{n}}$ "attached" at every point $\left(0, w_{2}, \ldots, w_{n}\right) \in D_{1}$ (this disc has only one common point with $D_{1}$ ).

Change coordinates on the $\operatorname{disc}\left\{w: w_{2}=\right.$ const, $\ldots, w_{n}=$ const $\}$ :

$$
\begin{gathered}
\left(\operatorname{Re} w_{1}, \operatorname{Im} w_{1}\right) \rightarrow(R, \Theta), \\
w_{1}=\sqrt{1-w_{2} \bar{w}_{2}-\ldots-w_{n} \bar{w}_{n}} R e^{i \Theta}, 0 \leq R \leq 1,0 \leq \Theta<2 \pi .
\end{gathered}
$$


Then on the $\operatorname{disc}\left\{w: w_{2}=\right.$ const, $\ldots w_{n}=$ const $\}$ :

$$
\begin{aligned}
d w_{1} \wedge d \bar{w}_{1} & =-2 i d\left(\operatorname{Re} w_{1}\right) \wedge d\left(\operatorname{Im} w_{1}\right) \\
=-2 i\left|\frac{\partial\left(\operatorname{Re} w_{1}, \operatorname{Im} w_{1}\right)}{\partial(R, \Theta)}\right| d R \wedge d \Theta & =-2 i\left(1-w_{2} \bar{w}_{2}-\ldots-w_{n} \bar{w}_{n}\right) R d R \wedge d \Theta, \\
d \Theta & =\left.\frac{1}{i} \frac{d w_{1}}{w_{1}}\right|_{R=\text { const } .}
\end{aligned}
$$

We have

$$
\begin{gathered}
-\langle w, w\rangle=\left(1-w_{2} \bar{w}_{2}-\ldots-w_{n} \bar{w}_{n}\right)\left(1-R^{2}\right), \\
-\left.\langle w, w\rangle\right|_{D_{1}}=1-w_{2} \bar{w}_{2}-\ldots-w_{n} \bar{w}_{n},
\end{gathered}
$$

SO

$$
I=-2 i(2 i)^{n} \alpha^{-(n+1) \kappa} \int_{0}^{1} R\left(1-R^{2}\right)^{2(n+1) \kappa-(n+1)} d R \frac{1}{i} \oint_{R=\text { const }} \frac{F\left(w_{1}\right)}{w_{1}} d w_{1},
$$

where

$$
\begin{aligned}
& F\left(w_{1}\right) \\
& =\int_{D_{1}} f(T w) \frac{\left(1-w_{2} \bar{w}_{2}-\ldots-w_{n} \bar{w}_{n}\right)^{2(n+1) \kappa-(n+1)+1}}{\left(1-\bar{w}_{n}^{2}\right)^{(n+1) \kappa}} j(T, w)^{2(n+1) \kappa} d V_{2}
\end{aligned}
$$

is a holomorphic function of $w_{1}$.

By the Cauchy integral formula

$$
\oint_{R=\text { const }} \frac{F\left(w_{1}\right)}{w_{1}} d w_{1}=2 \pi i F(0),
$$

hence this integral does not depend on the value of $R$ and, taking into account that $\left.\int_{0}^{1} R\left(1-R^{2}\right)^{2(n+1) \kappa-(n+1)} d R=\frac{1}{2(2(n+1) \kappa-(n+1)+1)}\right)$, we get:

$$
\begin{aligned}
I & =-2 i(2 i)^{n} \alpha^{-(n+1) \kappa} \frac{1}{i} 2 \pi i \frac{1}{2(2(n+1) \kappa-(n+1)+1)} \int_{D_{1}} f(T w) \\
& \frac{\left(1-w_{2} \bar{w}_{2}-\ldots-w_{n} \bar{w}_{n}\right)^{2(n+1) \kappa-(n+1)+1}}{\left(1-\bar{w}_{n}^{2}\right)^{(n+1) \kappa}} \operatorname{det} J(T, w)^{2 \kappa} d V_{2} \\
& =-\frac{2 \pi i}{2(n+1) \kappa-(n+1)+1} i^{n} \alpha^{-(n+1) \kappa} \times \\
& \int_{D_{1}} f(T w) \frac{(-\langle w, w\rangle)^{2(n+1) \kappa-(n+1)+1}}{\left(1-\bar{w}_{n}^{2}\right)^{(n+1) \kappa}} \operatorname{det} J(T, w)^{2 \kappa} d V_{2} .
\end{aligned}
$$

Step 2. Denote $D_{p}=T^{-1} F_{0} \cap\left\{w: w_{1}=0=\cdots=w_{p}=0\right\}$. $\gamma$ leaves invariant $\left\{w: w_{1}=\cdots=w_{p}=0\right\}$ for any $1 \leq p \leq n-1$. Repeat Step $1 n-2$ times more (i.e. totally we perform Step $1 n-1$ times). We 
prove that

$$
\begin{aligned}
& I=(2 i)^{n} \alpha^{-(n+1) \kappa}(-2 \pi i)^{p} \\
& \quad \frac{1}{2(n+1) \kappa-(n+1)+1} \cdots \frac{1}{2(n+1) \kappa-(n+1)+p} \\
& \quad \int_{D_{p}} f(T w) \frac{(-\langle w, w\rangle)^{2(n+1) \kappa-(n+1)+p}}{\left(1-\bar{w}_{n}^{2}\right)^{(n+1) \kappa}} j(T, w)^{2(n+1) \kappa} d V_{p+1} .
\end{aligned}
$$

This is proved by induction, and the step $p-1 \rightarrow p$ essentially repeats the argument in Step 1.

For $p=n-1$ we obtain

$$
\begin{aligned}
& I=(2 i)^{n} \alpha^{-(n+1) \kappa}(-2 \pi i)^{n-1} \\
& \quad \frac{1}{2(n+1) \kappa-(n+1)+1} \cdots \frac{1}{2(n+1) \kappa-(n+1)+n-1} \\
& \quad \int_{D_{n-1}} f(T w) \frac{(-\langle w, w\rangle)^{2(n+1) \kappa-(n+1)+n-1}}{\left(1-\bar{w}_{n}^{2}\right)^{(n+1) \kappa}} j(T, w)^{2(n+1) \kappa} d w_{n} \wedge d \bar{w}_{n} \\
& \quad=2^{n} i \alpha^{-(n+1) \kappa} \frac{(2(n+1) \kappa-n-1) !}{(2(n+1) \kappa-2) !}(2 \pi)^{n-1} \\
& \quad \int_{D_{n-1}} f(T w) \frac{(-\langle w, w\rangle)^{2(n+1) \kappa-2}}{\left(1-\bar{w}_{n}^{2}\right)^{(n+1) \kappa}} j(T, w)^{2(n+1) \kappa} d w_{n} \wedge d \bar{w}_{n} .
\end{aligned}
$$

Step 3. The last integral is over $D_{n-1}=T^{-1} F_{0} \cap\left\{w: w_{1}=0=\right.$ $\left.\cdots=w_{n-1}=0\right\}$, the fundamental domain for a "standard hyperbolic element"

$$
\gamma=\left(\begin{array}{ll}
\frac{\lambda^{2}+1}{2 \lambda} & \frac{\lambda^{2}-1}{2 \lambda} \\
\frac{\lambda^{2}-1}{2 \lambda} & \frac{\lambda^{2}+1}{2 \lambda}
\end{array}\right)
$$

of $S U(1,1)$ acting on the unit disc in $\mathbb{C}$. The change of coordinates

$$
\left(\operatorname{Re} w_{n}, \operatorname{Im} w_{n}\right) \rightarrow(r, \phi),(r>0,0<\phi<\pi)
$$

where

$$
w_{n}=\frac{u-i}{u+i}, \quad u=r e^{i \phi}
$$

maps the unit disc to the upper half-plane, and by a standard technique 18 we obtain 


$$
\begin{aligned}
I & =2^{n-1} \alpha^{-(n+1) \kappa} \frac{(2(n+1) \kappa-n-1) !}{(2(n+1) \kappa-2) !}(2 \pi)^{n-1} \int_{0}^{\pi}(\sin \phi)^{2(n+1) \kappa-2} d \phi \times \\
& \int_{|\lambda|^{-1}}^{|\lambda|} f(T w)\left(1-w_{n}^{2}\right)^{(n+1) \kappa} j(T, w)^{2(n+1) \kappa} \frac{1}{r} d r \\
& =2^{n-1} \alpha^{-(n+1) \kappa} \frac{(2(n+1) \kappa-n-1) !}{(2(n+1) \kappa-2) !}(2 \pi)^{n-1} \int_{0}^{\pi}(\sin \phi)^{2(n+1) \kappa-2} d \phi \times \\
& 2 \int_{w_{n}}^{\gamma w_{n}} f(T w)\left(1-w_{n}^{2}\right)^{(n+1) \kappa} j(T, w)^{2(n+1) \kappa} \frac{d w_{n}}{1-w_{n}^{2}},
\end{aligned}
$$

The last integral does not depend on $\phi$ as the integral of a $\gamma$-invariant holomorphic function of $w_{n}$. For the same reason it does not depend the choice of the point $w_{0}$ and the path from $w_{0}$ to $\gamma w_{0}$ in $D_{n-1}$. Hence we may take $w_{n}, \gamma w_{n} \in \mathfrak{I}$, and since on $\mathfrak{I} d t=\frac{d w_{n}}{1-w_{n}^{2}}$, and the last integral can be rewritten as

$$
\int_{w_{0}}^{\gamma w_{0}} f(T w)\left(1-w_{n}^{2}\right)^{(n+1) k} j(T, w)^{2(n+1) \kappa} d t .
$$

where the integration is over a segment of $\mathfrak{I}$.

Using Lemma 12 again, we go back to the integral over the axis of $\gamma_{0}$, and obtain

$$
\begin{aligned}
I= & 2^{n}|\alpha|^{-2(n+1) \kappa} \frac{(2(n+1) \kappa-n-1) !}{(2(n+1) \kappa-2) !}(2 \pi)^{n-1} \\
& \int_{0}^{\pi}(\sin \phi)^{2(n+1) \kappa-2} d \phi \int_{z_{0}}^{\gamma_{0} z_{0}} f(z)(\langle z, X\rangle\langle z, Y\rangle)^{(n+1) \kappa} d t,
\end{aligned}
$$

Finally we obtain

$$
\left(f, \Theta_{\gamma_{0}, \kappa}\right)=|\alpha|^{-2(n+1) \kappa} C \int_{z_{0}}^{\gamma_{0} z_{0}} f(z)(\langle z, X\rangle\langle z, Y\rangle)^{(n+1) \kappa} d t
$$

with

$$
C=\frac{(2(n+1) \kappa-n-1) !}{(((n+1) \kappa-1) !)^{2}} \pi^{n} 2^{2(n+1)(1-\kappa)-1}
$$

The following Corollary is immediate from Theorem 11.

Corollary 13. Let $\gamma_{1}, \gamma_{0} \in \Gamma$ be two primitive loxodromic elements having the same axis. Then $\Theta_{\gamma_{0}, \kappa}=\Theta_{\gamma_{1}, \kappa}$.

The integral (35) is well-defined and is called the period of $f$ over the closed geodesic $\left[g_{0}\right]$. The reason for this definition is the following result. 
Theorem 14. Let $\tilde{f}$ be the lift of the cusp form $f(z) \in S_{2(n+1) \kappa}(\Gamma)$ to $G$. Then for any lift $\left[\gamma_{0}\right]_{w}$ of the closed geodesic $\left[\gamma_{0}\right]$ to $G$ we have

Here $w=\left(\begin{array}{ccc}u_{n-1} & 0 & 0 \\ 0 & e^{-i \psi} & 0 \\ 0 & 0 & e^{-i \psi}\end{array}\right)$ with $\operatorname{det} u_{n-1}=e^{2 i \psi}$, and $C$ is the constant from Theorem 11.

Proof. We make a change of variables $g$ used in $\$ 2.1$ which maps the "horizontal" geodesic to the axis of $\gamma_{0}$ in such a way that $g(0)=z_{0}$. According to $\$ 2.1$ all lifts of the segment of the geodesic $\left[z_{0}, \gamma_{0} z_{0}\right]$ are given by $\left\{g w a_{t} \mid w \in W, 0 \leq t \leq s\right\}$, and all lifts of the corresponding segment of the "horizontal" geodesic, by $\left\{w a_{t} \mid w \in W, 0 \leq t \leq s\right\}$. Using local coordinates (31) on $G$ we obtain

$$
\begin{aligned}
& \int_{z_{0}}^{\gamma_{0} z_{0}} f(z)(\langle z, X\rangle\langle z, Y\rangle)^{(n+1) \kappa} d t \\
= & \bar{\alpha}^{(n+1) \kappa} \int_{0}^{g^{-1} \gamma_{0} g(0)} f(g x)\left(1-x_{n}^{2}\right)^{(n+1) \kappa} j(g, x)^{2(n+1) \kappa} d t \\
= & \bar{\alpha}^{(n+1) \kappa} \int_{\left[g^{-1} \gamma_{0} g\right]_{w}} f(g x)\left(e^{-i \psi} \zeta_{x}\right)^{2(n+1) \kappa} j(g, x)^{2(n+1) \kappa} d t \\
= & \bar{\alpha}^{(n+1) \kappa} e^{-2(n+1) \kappa i \psi} \int_{\left[\gamma_{0}\right]_{w}} f(z) \zeta_{z}^{2(n+1) \kappa} d t .
\end{aligned}
$$

The last two integrals are in $G$ over the lifts $\left[g^{-1} \gamma_{0} g\right]_{w}$ and $\left[\gamma_{0}\right]_{w}$, and $x$, $\zeta_{x}$ and $z, \zeta_{z}$ are evaluated at the corresponding value of the parameter $t: x_{n}=\left(0, \ldots, x_{n}(t)\right)=\varphi_{t}(0), x_{n}(t)=\tanh t, \zeta_{x}=\frac{e^{i \psi}}{\cosh t}$. We used the cocycle identity to obtain the last equality:

$$
\zeta_{z}=j(g, x) \zeta_{x} .
$$

The required formula now follows from Theorem 11. Notice that since $\tilde{f}=f(z) \zeta_{z}^{2(n+1) \kappa}$ is $\Gamma$-invariant, the integral is in $\Gamma \backslash G$.

\section{Cohomological Equation For CUSP Forms: the VANishing RESULT}

8.1. Three-dimensional subalgebra. Now we consider $L^{2}(\Gamma \backslash G)$ with the inner product (7). The infinitesimal generators of the frame flow (28) and the one-parameter subgroup

$$
m_{\psi}=\left\{\left(\begin{array}{ccc}
1_{n-1} & 0 & 0 \\
0 & e^{i \psi} & 0 \\
0 & 0 & e^{-i \psi}
\end{array}\right) \mid \psi \in \mathbb{R} / 2 \pi \mathbb{Z}\right\} \subset K
$$


belong to the Lie algebra $\mathfrak{g}=\mathfrak{s u}(n, 1)$. The corresponding left-invariant differential operators

$$
\mathfrak{D} F(g)=\left.\frac{d}{d t} F\left(g \cdot a_{t}\right)\right|_{t=0} \text { and } \frac{\partial}{\partial \psi} F(g)=\left.\frac{\partial}{\partial \psi} F\left(g \cdot m_{\psi}\right)\right|_{\psi=0}
$$

are defined on a dense set of functions in $L^{2}(\Gamma \backslash G)$ differentiable along the orbits of the corresponding flows, and are given by the matrices

$$
\mathfrak{D}=\left(\begin{array}{ccc}
0_{n-1} & 0 & 0 \\
0 & 0 & 1 \\
0 & 1 & 0
\end{array}\right), \frac{\partial}{\partial \psi}=\left(\begin{array}{ccc}
0_{n-1} & 0 & 0 \\
0 & i & 0 \\
0 & 0 & -i
\end{array}\right) \text {. }
$$

Complemented by the third differential operator

$$
\mathfrak{D}^{\prime}=\left(\begin{array}{ccc}
0_{n-1} & 0 & 0 \\
0 & 0 & i \\
0 & -i & 0
\end{array}\right)
$$

they generate a three-dimensional Lie subalgebra of $\mathfrak{g}$ with the commutation relations:

$$
\left[\frac{\partial}{\partial \psi}, \mathfrak{D}\right]=2 \mathfrak{D}^{\prime},\left[\frac{\partial}{\partial \psi}, \mathfrak{D}^{\prime}\right]=-2 \mathfrak{D},\left[\mathfrak{D}, \mathfrak{D}^{\prime}\right]=-2 \frac{\partial}{\partial \psi} .
$$

Then

$$
\mathfrak{D}^{+}=\frac{\mathfrak{D}-i \mathfrak{D}^{\prime}}{2}, \mathfrak{D}^{-}=\frac{\mathfrak{D}+i \mathfrak{D}^{\prime}}{2} \text { and } \Psi=-i \frac{\partial}{\partial \psi}
$$

belong to the complexification of $\mathfrak{g}, \mathfrak{g}^{c}=\mathfrak{s l}(n+1, \mathbb{C})$ and have the following commutation relations:

$$
\left[\Psi, \mathfrak{D}^{+}\right]=2 \mathfrak{D}^{+},\left[\Psi, \mathfrak{D}^{-}\right]=-2 \mathfrak{D}^{-},\left[\mathfrak{D}^{+}, \mathfrak{D}^{-}\right]=\Psi .
$$

They generate a three-dimensional real Lie algebra isomorphic to $\mathfrak{s l}(2, \mathbb{R})$. The properties of these operators are exactly the same as in the case $n=1(\operatorname{cf}[8], \S 3$, [12], 22$)$.

Proposition 15. The differential operator $\mathfrak{D}$ is skew-self-adjoint: $\mathfrak{D}^{*}=$ - $\mathfrak{D}$, or equivalently, $(\mathfrak{D} F, H)=-(F, \mathfrak{D} H)$ for $F, H \in L^{2}(\Gamma \backslash G) \cap$ domain $\mathfrak{D}$.

A standard Fourier analysis argument shows that the space $L^{2}(\Gamma \backslash G)$ can be decomposed into a direct sum of orthogonal subspaces $\sum H_{m}$ such that

$$
H_{m}=\left\{F \in L^{2}(\Gamma \backslash G) \mid \Psi F=m F\right\} .
$$

Notice that the lift of a cusp form $f \in S_{(n+1) \kappa}(\Gamma)$ to the group $G$ belongs to the space $H_{(n+1) \kappa}$. 
Proposition 16. 1. If $F \in H_{m}$, then $\mathfrak{D}^{+} F \in H_{m+2}$, and $\mathfrak{D}^{-} F \in$ $H_{m-2}$;

2. $\left(\mathfrak{D}^{+}\right)^{*}=-\mathfrak{D}^{-} ;\left(\mathfrak{D}^{-}\right)^{*}=-\mathfrak{D}^{+}$.

8.2. Proof of Theorem 1. Suppose there is a cusp form $f \in S_{2(n+1) \kappa}(\Gamma)$, such that $\left(f, \Theta_{\gamma_{0}, \kappa}\right)=0$ for all loxodromic elements $\gamma_{0} \in \Gamma$. Then by Theorem 14 the function $f(z) \zeta^{2(n+1) \kappa}$ satisfies the Theorem 8, and its application guarantees us existence of a smooth function $F: \Gamma \backslash G \rightarrow \mathbb{C}$ such that

$$
\mathfrak{D} F=f(z) \zeta^{2(n+1) \kappa} .
$$

Lemma 17. Let $F$ be the function obtained from the Theorem 8 for $f(z) \zeta^{2(n+1) \kappa}$, where $f(z) \in S_{\mu}(\Gamma)$. Then $F \in L^{2}(\Gamma \backslash G)$.

Proof. The uniform boundedness of $|F|$ on $\Gamma \backslash G$ of finite volume would imply the required result. If $\Gamma \backslash G$ is compact, the uniform boundedness follows from the Lipschitz condition. Alternatively, $\Gamma \backslash G$ has a finite number of cusps [5], and it is sufficient to show that $|F|$ is bounded at each cusp $\sigma$. The proof is similar to the proof for Fuchsian groups in 12. It is based on an application of a partial Cayley transform $R$ mapping a Siegel domain in $\mathcal{S} \in \mathbb{C}^{n}$ to $B^{n}=\left\{\sum_{i=1}^{n}\left|z_{i}\right|^{2}<1\right\}$ in such a way that $R(\infty)=\sigma$. Since the real rank of $G$ is equal to one, we have

$\mathcal{S}=\left\{(z, u)=\left.\left(z, u_{1}, \ldots, u_{n-1}\right) \in \mathbb{C}^{n}|\operatorname{Im} \mathrm{z}-| \mathrm{u}_{1}\right|^{2}-\cdots-\left|\mathrm{u}_{\mathrm{n}-1}\right|^{2}>0\right\}$.

If $\Gamma_{\sigma}=\{\gamma \in \Gamma \mid \gamma(\sigma)=\sigma\}$, then $R^{-1} \Gamma_{\sigma} R$ consists of the "parallel translations" $T_{m}:(z, u) \rightarrow(z+m, u),(m \in \mathbb{Z})$, with $j\left(T_{m},(z, u)\right)=1$, and $\Phi(z, u)=(f \mid R)(z, u)=f(R(z, u)) j(R,(z, u))^{2(n+1) \kappa}$ is invariant under $T_{m}: \Phi(z+m, u)=\Phi(z, u)$. Then $\Phi$ has a Fourier-Jacobi expansion (22], Ch. 3, §5, [2], Ch. 11)

$$
\Phi(z, u)=\sum_{m \in \mathbb{Z}} \psi_{m}(u) e^{2 \pi i m z} .
$$

Since $f$ is a cusp form, $\psi_{m}(u)=0$ for $m \leq 0$. Let $I$ be the geodesic in $\mathcal{S}$ given by $\operatorname{Rez}=0, u=0$. Then $R(I)$ is the geodesic on $B^{n}$ going to the cusp $\sigma$. On $I$ we have $|\Phi(z, u)|=O\left(e^{-2 \pi y}\right)$, where $y=\operatorname{Im} z$. Thus $\mid F\left(\varphi_{s}(v)-F(v) \mid\right.$ may be estimated by the integral $\int_{y_{0}}^{\infty}|\Phi(z, u)| y^{(n+1) \kappa} \frac{d y}{y}$ over $I$, which is finite. The finiteness of the volume implies that for any $\epsilon>0$ there exists a neighborhood of the cusp $U(\sigma)$ such that $d((z, u), R(I))<\epsilon$, and the uniform boundedness of $|F|$ now follows from the Lipschitz condition. 
We decompose $F$ according to (37) and rewrite (38) as the following system

$$
\begin{aligned}
& \mathfrak{D}^{-} F_{2(n+1) \kappa+2}+\mathfrak{D}^{+} F_{2(n+1) \kappa-2}=f(z) \zeta^{2(n+1) \kappa} \\
& \mathfrak{D}^{-} F_{j+2}+\mathfrak{D}^{+} F_{j-2}=0 \text { for all } j \neq 2(n+1) \kappa
\end{aligned}
$$

The argument of Guillemin and Kazhdan ( 8 , Th. 3.6) for negatively curved surfaces is applicable to this situation since it depends only on the commutation relations (36) and the fact which immediately follows from it: for $F_{m} \in H_{m}$

$$
\left\|\mathfrak{D}^{+} F_{m}\right\|^{2}=\left\|\mathfrak{D}^{-} F_{m}\right\|^{2}+m\left\|F_{m}\right\|^{2} .
$$

Proposition 18. Let $F$ be a solution of (39). Then $F_{j}=0$ for $j \geq$ $2(n+1) \kappa$.

Thus $F_{2(n+1) \kappa+2}=0$ and the first equation of (39) has the form

$$
\mathfrak{D}^{+} F_{2(n+1) \kappa-2}=f(z) \zeta^{2(n+1) \kappa}
$$

Proposition 19. If $\tilde{f}(g)=f(z) \zeta^{2(n+1) \kappa}$ is the lift of a holomorphic cusp form $f \in S_{2(n+1) \kappa}$ to $G$, then $\mathfrak{D}^{-} f(z) \zeta^{2(n+1) \kappa}=0$.

Proof. It follows immediately from the fact that has been, apparently, first pointed out in [6] (see also [1], $\S 5$ and [3] p. 203) that for any $Y \in \mathfrak{p}^{-}$

$$
Y \tilde{f}(g)=j(g, 0)^{2(n+1) \kappa}(\tilde{Y} f)(z) .
$$

Here $\tilde{Y}$ is the linear combination of the partial derivatives $\frac{\partial}{\partial \bar{z}_{j}}$ where $z_{j}$ are coordinates on the bounded domain $B^{n}$ and $z=g(0)=\left(z_{1}, \ldots, z_{n}\right)$. For $Y=\mathfrak{D}^{-} \in \mathfrak{p}^{-}$this can be easily checked by a direct differentiation along the orbit of the frame flow $\tilde{\varphi}_{t}(g)$ using the decomposition $g=$ $g_{0} \cdot m_{\psi}$ corresponding to the local coordinates (31) with coefficients of

$$
\begin{aligned}
& g_{0}=\left(\begin{array}{cccc}
a_{11} & \ldots & a_{1 n} & b_{1} \\
\ldots & \ldots & \ldots & \ldots \\
a_{n 1} & \ldots & a_{n n} & b_{n} \\
c_{1} & \ldots & c_{n} & d
\end{array}\right) \text { independent on } \psi \text {. Then } \\
& \mathfrak{D}\left(f(z, \bar{z}) \zeta^{m}\right) \\
& =\left(\sum\left(a_{j n} d-c_{n} b_{j}\right) \frac{\partial f}{\partial z_{j}}-m c_{n} d f(z, \bar{z})\right) \zeta^{m+2} \\
& +\left(\sum \frac{\overline{\left(a_{j n} d-c_{n} b_{j}\right)}}{(d \bar{d})^{2}} \frac{\partial f}{\partial \bar{z}_{j}}\right) \zeta^{m-2} .
\end{aligned}
$$


Since $f(z)$ is holomorphic we have

$$
\mathfrak{D}^{-} f(z) \zeta^{2(n+1) \kappa}=\left(\sum \frac{\overline{\left(a_{j n} d-c_{n} b_{j}\right)}}{(d \bar{d})^{2}} \frac{\partial f}{\partial \bar{z}_{j}}\right) \zeta^{2(n+1) \kappa-2}=0 .
$$

The end of the proof goes exactly as in [12]. By Proposition 19

$$
\mathfrak{D}^{-} \mathfrak{D}^{+} F_{2(n+1) \kappa-2}=\mathfrak{D}^{-} f(z) \zeta^{2(n+1) \kappa}=0 .
$$

Therefore

$$
0=\left(F_{2(n+1) \kappa-2}, \mathfrak{D}^{-} \mathfrak{D}^{+} F_{2(n+1) \kappa-2}\right)=-\left\|\mathfrak{D}^{+} F_{2(n+1) \kappa-2}\right\|^{2},
$$

hence $f(z) \zeta^{2(n+1) \kappa-2}=\mathfrak{D}^{+} F_{2(n+1) \kappa-2}=0$. Since $\zeta \neq 0, f(z)=0$.

\section{REFERENCES}

[1] W. Baily and A. Borel, Compactification of arithmetic quotients of bounded symmetric domains, Ann. Math. 84 (1966), 442-528

[2] W. Baily, Introductory lectures on automorphic forms, Publ. Math. Soc. Japan 12 (1973), I. Shoten and Princeton Univ. Press.

[3] A. Borel, Introduction to automorphic forms, Proc. Symp. Pure Math. 9 (1966), 199-210

[4] P. Eberlein, Geometry of nonpositively curved manifolds, University of Chicago Press, Chicago, 1996

[5] H. Garland and M.S. Raghunathan, Fundamental domains for lattices in (R-)rank 1 semisimple Lie groups, Ann. Math., Second Series, 92, No. 2 (1970), 279-326

[6] R. Godement, Série Poincaré et Spitzenformen, Séminaire H. Cartan, E.N.S., 10ieme année, 1957-58

[7] W. M. Goldman, Complex hyperbolic geometry, Oxford University Press, Oxford, England, 1999

[8] V. Guillemin and D. Kazhdan, Some inverse spectral results for negatively curved 2-manifolds, Topology 19, (1980) 301-312

[9] S. Helgason, Differential geometry, Lie groups, and symmetric spaces, Academic Press, New York, 1978

[10] Harish-Chandra, Automorphic forms on a semi-simple Lie group, Proc. Nat. Acad. Sci. U.S.A. 45 (1959), 570-573

[11] Harish-Chandra, Automorphic forms on semi-simple Lie groups, Lecture Notes in Math. 62 Springer Verlag, New York, 1968

[12] S. Katok, Closed geodesics, periods and arithmetic of modular forms, Invent. Math. 80 (1985), 469-480

[13] S. Katok, Modular forms associated to closed geodesics and arithmetic applications, Ph.D. Thesis, University of Maryland (1983)

[14] S. Katok, Finite spanning sets for cusp forms and a related geometric result, J. reine angew. Math. 395 (1989), 186-195

[15] S. Katok, Approximate solutions of cohomological equations associated with some Anosov flows, Ergod. Th. \& Dynam. Sys. 10 (1990), 367-379 
[16] A. Katok and B. Hasselblatt, Introduction to the modern theory of dynamical systems, Encyclopaedia of Mathematics and its Applications, 54, Cambridge University Press, New York, 1995

[17] D.A. Kazhdan, Connection of the dual space of a group with the structure of its closed subgroups, Functional Anal. Appl. (A translation of Funk. Anal. i Pril.) 1 (1967), 63-65

[18] W. Kohnen, Beziehungen zwischen Modulformen halbzangen Gewichts und Modulformen ganzen Gewichts. Bonner Mathematische Schriften 131, Bonn (1981)

[19] S. Kobayashi and K. Nomizu, Foundations of differential geometry, II, John Wiley \& Sons, New York, 1969

[20] A. Livshitz, Some homological properties of U-systems, Mat. Zametki 10 (1971), 555-564

[21] G.A. Margulis, Discrete subgroups of semisimple Lie groups, Springer Verlag, Berlin-Heidelberg-New York, 1991

[22] I.I.Pyatetskii-Shapiro, Automorphic functions and the geometry of classical domains, Gordon and Breach, New York - London-Paris, 1969 (Russian edition: Fizmatgiz, 1961)

[23] H. Reznikoff and Y.-S. Tai, On the structure of a graded ring of automorphic forms on the 2-dimensional complex ball, Math. Ann. 238 (1987), 97-117

[24] Séminaire H.Cartan, E.N.S., 1957-58, mimeographic notes

[25] R. Zimmer, Ergodic theory and semisimple groups, Monographs in Mathematics, 81, Birkhaüser, Boston-Basel-Stuttgart, 1984

Department of Mathematics, Pennsylvania State University, UniVERSITY PARK, PA 16802

E-mail address: t_foth@math.psu.edu

Department of Mathematics, Pennsylvania State University, UniVERSITY PARK, PA 16802

E-mail address: katok_s@math.psu.edu 